\title{
A Solvable Mixed Charge Ensemble on the Line: Global Results
}

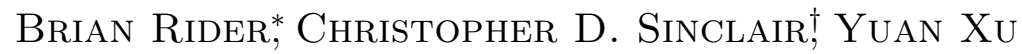

November 19, 2018

\begin{abstract}
We consider an ensemble of interacting charged particles on the line consisting of two species of particles with charge ratio $2: 1$ in the presence of the harmonic oscillator potential. The system is assumed to be at temperature corresponding to $\beta=1$ and the sum of the charges is fixed. We investigate the distribution of the number as well as the spatial density of each species of particle in the limit as the total charge increases to $\infty$. These results will follow from the fact that the system of particles forms a Pfaffian point process. We produce the skew-orthogonal polynomials necessary to simplify the related matrix kernels.
\end{abstract}

\section{Introduction}

Let $L, M$ and $N$ be non-negative integers so that $L+2 M=N$, and consider 1-dimensional electrostatic system consisting of $L$ particles with unit charge and $M$ particles with charge 2 . We will identify the state of the system by pairs of finite subsets of $\mathbb{R}, \xi_{1}=\left\{\alpha_{1}, \alpha_{2}, \ldots, \alpha_{L}\right\}$ and $\xi_{2}=\left\{\beta_{1}, \beta_{2}, \ldots \beta_{M}\right\}$, where $\alpha_{1}, \alpha_{2}, \ldots, \alpha_{L}$ represent the locations of the charge 1 particles and $\beta_{1}, \beta_{2}, \ldots, \beta_{M}$ represent the locations of the charge 2 particles.

The potential energy of state $\xi=\left(\xi_{1}, \xi_{2}\right)$ is given by

$$
\sum_{j<k} \log \left|\alpha_{j}-\alpha_{k}\right|+4 \sum_{m<n} \log \left|\beta_{m}-\beta_{n}\right|+2 \sum_{\ell=1}^{L} \sum_{m=1}^{M}\left|\alpha_{\ell}-\beta_{m}\right| .
$$

We assume that the system is in the presence of an external field, so that the interaction energy between the charges and the field is given by

$$
-\sum_{\ell=1}^{L} V\left(\alpha_{\ell}\right)-2 \sum_{m=1}^{M} V\left(\beta_{m}\right)
$$

for some potential $V: \mathbb{R} \rightarrow[0, \infty)$. Eventually we will specify to the situation where $V$ is the harmonic oscillator potential, but for now we maintain generality. The total potential

* partially supported by NSF grant DMS-0645756.

${ }^{\dagger}$ partially supported by NSF grant DMS-0801243 
energy of the system is therefore

$$
\begin{aligned}
E=\sum_{j<k} \log \left|\alpha_{j}-\alpha_{k}\right|+4 \sum_{m<n} \log \left|\beta_{m}-\beta_{n}\right| & +2 \sum_{\ell=1}^{L} \sum_{m=1}^{M} \log \left|\alpha_{\ell}-\beta_{m}\right| \\
& -\sum_{\ell=1}^{L} V\left(\alpha_{\ell}\right)-2 \sum_{m=1}^{M} V\left(\beta_{m}\right) .
\end{aligned}
$$

Given a pair of vectors $(\boldsymbol{\alpha}, \boldsymbol{\beta}) \in \mathbb{R}^{L} \times \mathbb{R}^{M}$ we will define $E(\boldsymbol{\alpha}, \boldsymbol{\beta})$ to be the right hand side of (1.1), and call $(\boldsymbol{\alpha}, \boldsymbol{\beta})$ a state vector corresponding to the state $\xi$. Generically, there are $L ! M !$ state vectors corresponding to a given state.

Assuming the system is placed in a heat bath corresponding to inverse temperature parameter $\beta=1$, then the Boltzmann factor for the state vector $(\boldsymbol{\alpha}, \boldsymbol{\beta})$ is given by

$$
e^{-E(\boldsymbol{\alpha}, \boldsymbol{\beta})}=\prod_{\ell=1}^{L} w\left(\alpha_{\ell}\right) \prod_{m=1}^{M} w\left(\beta_{m}\right)^{2} \prod_{j<k}\left|\alpha_{j}-\alpha_{k}\right| \prod_{m<n}\left|\beta_{m}-\beta_{n}\right|^{4} \prod_{\ell=1}^{L} \prod_{m=1}^{M}\left|\alpha_{\ell}-\beta_{m}\right|^{2},
$$

where $w(\gamma)=e^{-V(\gamma)}$ is the weight of the system. The partition function of the system is given by

$$
Z_{L, M}=\frac{1}{L ! M !} \int_{\mathbb{R}^{L}} \int_{\mathbb{R}^{M}} e^{-E(\boldsymbol{\alpha}, \boldsymbol{\beta})} d \mu^{L}(\alpha) d \mu^{M}(\beta),
$$

where $\mu$ and $\mu^{L}$ are Lebesgue measure on $\mathbb{R}$ and $\mathbb{R}^{L}$ respectively. The multiplicative prefactor $1 /(L ! M !)$ compensates for the multitude of state vectors associated to each state.

Here we will be interested in a form of the grand canonical ensemble conditioned so that the sum of the charges equals $N$. That is, we consider the union of all two component ensembles with $L$ particles of charge 1 and $M$ particles of charge 2 over all pairs of nonnegative integers $L$ and $M$ with $L+2 M=N$. The partition function of this ensemble is given by

$$
Z(X)=\sum_{(L, M)} X^{L} Z_{L, M}=\sum_{(L, M)} \frac{X^{L}}{L ! M !} \int_{\mathbb{R}^{L}} \int_{\mathbb{R}^{M}} e^{-E(\boldsymbol{\alpha}, \boldsymbol{\beta})} d \mu^{L}(\alpha) d \mu^{M}(\beta) .
$$

Here $X \geq 0$ is the fugacity of the system, a parameter which controls the probability that the system has a particular population vector $(L, M)$. The sum over $(L, M)$ indicates that we are summing over all pairs of non-negative integers such that $L+2 M=N$.

Note now that $(L, M)$ is itself a random vector, though we will continue to use this notation for the value of the population vector as well. For example, for each admissible pair $(L, M)$, the joint density of particles given population vector $(L, M)$ is given by the normalized Boltzmann factor,

$$
\frac{X^{L}}{Z(X)} e^{-E(\boldsymbol{\alpha}, \boldsymbol{\beta})}
$$

When $X=1$ the probability of seeing a particular pair $(L, M)$, or $\operatorname{Prob}(L, M)$, is the ratio $Z_{L, M} / Z$, where $Z=Z(1)$.

Experts of random matrix theory will have already noticed that when $X=0$ the above reduces to a general orthogonal (or $\beta=1$ ) ensemble. Likewise, as $X \rightarrow \infty$, the above formally goes over to the corresponding symplectic (or $\beta=4$ ) ensemble. This provides then an unusual sort of interpolation between two classical and well studied point processes. 


\section{Statement of results}

In this paper we will primarily be concerned with global statistics of the particles when the fugacity equals 1 and the potential $V$ is given by

$$
V(\gamma)=\gamma^{2} / 2, \quad \text { that is, } \quad w(\gamma)=e^{-\gamma^{2} / 2} .
$$

Many of the results presented here are valid for other potentials and other values of $X$, however unless otherwise indicated we will restrict ourselves to these choices of $V$ and $X$. We will also restrict ourselves to the situation where $N=2 J$ is an even integer.

Similar results for the two-charge ensemble constrained to the circle with uniform weight were obtained by P.J. Forrester (see 5.9 of $[7$ and the references therein).

The goal of this paper is to present global results about the distribution of $L$ and $M$ as well as the global spatial distribution of each of the species of particles. Along the way we will derive a Pfaffian point process for the particles (similar to that of another twocomponent ensemble, Ginibre's real ensemble) as well as the skew-orthogonal polynomials which allow us to present a simplified matrix kernel for the process. The local analysis of this kernel (i.e. its scaling limits in the bulk and at the edge) as well an investigation of the right-most particle of each species will appear in a forthcoming publication.

\subsection{Distribution of the population vectors}

Sharp results on the law of the state vector $(L, M)$ are consequences of the following characterization.

Theorem 2.1. For each non-negative integer $j$, let $L_{j}=L_{j}^{(-1 / 2)}$ be the $j$ th Laguerre polynomial with parameter $\alpha=-1 / 2$. Then, $\operatorname{Prob}(L, M)$ is the coefficient of $X^{L}$ of the polynomial $L_{N / 2}\left(-X^{2}\right) / L_{N / 2}(-1)$. That is,

$$
\frac{Z(X)}{Z}=\frac{L_{N / 2}\left(-X^{2}\right)}{L_{N / 2}(-1)}
$$

and so

1. $\operatorname{Prob}(L, M)=\frac{2^{L}}{L ! M !} \sum_{\substack{\ell, m) \\ \ell+2 m=N}} \frac{2^{\ell}}{\ell ! m !} \quad$ if $L$ is even, and is equal to 0 otherwise,

2. $\mathbb{E}\left(L^{m}\right)=\left[\left(X \frac{d}{d X}\right)^{m} \frac{L_{N / 2}\left(-X^{2}\right)}{L_{N / 2}(-1)}\right]_{X=1} \quad$ for $m$ non-negative integer.

Properties of the Laguerre polynomials now allow for nice expressions for the mean, variance, etc. of $L$ for all finite values of $N$. For example, we have that

$$
\mathbb{E}[L]=\frac{d}{d X}\left[\frac{Z(X)}{Z}\right]_{X=1}=2 \sum_{j=0}^{N / 2-1} \frac{L_{j}(-1)}{L_{N / 2}(-1)}=\frac{2 \sum_{i=0}^{N / 2-1}\left[\Gamma\left(\frac{N}{2}-i\right) \Gamma\left(i+\frac{3}{2}\right) i !\right]^{-1}}{\sum_{i=0}^{N / 2}\left[\Gamma\left(\frac{N}{2}-i+1\right) \Gamma\left(i+\frac{1}{2}\right) i !\right]^{-1}} .
$$

Asymptotic descriptions of the law of $L$ are just as readily obtained from Theorem 2.1. 
Theorem 2.2. As $N \rightarrow \infty$ it holds:

1. $\mathbb{E}(L)=\sqrt{2 N}-1+\frac{1}{3 \sqrt{N}}+\mathrm{O}\left(N^{-1}\right)$ and $\operatorname{Var}(L)=\sqrt{2 N}-\frac{4}{3}+\mathrm{O}\left(N^{-1 / 2}\right)$,

2. $\frac{L-(2 N)^{1 / 2}}{(2 N)^{1 / 4}}$ converges in distribution to a standard Normal random variable,

3. $\operatorname{Prob}\left(\left|\frac{L}{\sqrt{2 N}}-1\right| \geq \epsilon\right) \leq C N e^{-(\epsilon \wedge 1) \sqrt{2 N}}$ with a numerical constant $C$ for any $\epsilon>0$.

\subsection{Spatial density of particles}

We introduce the (mean) counting measures $\rho_{1}$ and $\rho_{2}$ for the charge 1 and charge 2 particles defined by

$$
\mathbb{E}\left[\left|A \cap \xi_{1}\right|\right]=\int_{A} d \rho_{1} \quad \text { and } \quad \mathbb{E}\left[\left|A \cap \xi_{2}\right|\right]=\int_{A} d \rho_{2}
$$

for Borel subsets $A \subseteq \mathbb{R}$ (where, for instance, $\left|A \cap \xi_{1}\right|$ is the number of charge 1 particles in $A$ ). As we shall see in the sequel, these measures are absolutely continuous with respect to Lebesgue measure, and we will write $R_{1,0}^{(N)}(x)$ and $R_{0,1}^{(N)}(x)$ for their respective densities. (The cryptic notation will be resolved in Section 3.2, when we define the $\ell, m$-correlation function of the ensemble to be $R_{\ell, m}^{(N)}$ ).

From Theorem 2.2 we see that, as $N \rightarrow \infty$,

$$
\int_{-\infty}^{\infty} R_{1,0}^{(N)}(x) d x=\mathbb{E}[L] \sim \sqrt{2 N}, \quad \text { and } \quad \int_{-\infty}^{\infty} R_{0,1}^{(N)}(x) d x=\mathbb{E}[M] \sim \frac{N}{2} .
$$

One then would ask, when suitably scaled and normalized as in

$$
s_{1}^{(N)}(x)=\frac{1}{\sqrt{2}} R_{1,0}^{(N)}(\sqrt{N} x) \quad \text { and } \quad s_{2}^{(N)}(x)=\frac{2}{\sqrt{N}} R_{0,1}^{(N)}(\sqrt{N} x),
$$

whether $s_{1}^{(N)}(x) d x$ and $s_{2}^{(N)}(x) d x$ converge to proper probability measures. This is answered in the affirmative in Theorem 2.3 below.

The previous result shows that, with probability one, for all $N$ large the number of charge 1 particles is $\sqrt{2 N}(1+o(1))$. This suggests that, in the thermodynamic limit, the statistics of the charge 2 particles should behave as though there are no charge 1 particles present, or like a copy of the Gaussian Symplectic Ensemble (again, arrived at from the present ensemble upon setting $L=0$ ). Indeed we find the scaled density of charge 2 particles approaches the semi-circle law.

On the other hand, though the charge 1 particles exhibit the same level repulsion amongst themselves as the eigenvalues in the Gaussian Orthogonal Ensemble (occurring here when $M=0$ ), the preponderance of charge 2 particles leads to a different limit distribution.

Ginibre's real ensemble, the ensemble of eigenvalues of real asymmetric matrices with i.i.d. Gaussian entries, has superficial resemblance to the ensemble we are considering here. First, it is suggestive to think of the present ensemble as arising from real Ginibre by forcing the non-real eigenvalues, which occur in complex conjugate pairs, to be identified with one "charge two" particle on the line. A little more concretely, the (random) number of real eigenvalues in real Ginibre has both expectation and variance of $O(\sqrt{N})$, as does the number of charge 1 particles here. (See [6] for the mean, and $[\underline{8}$ for the variance). It is perhaps not surprising, therefore, that the limiting scaled density of charge 1 particles is the same (up to a constant) as that of the real eigenvalues in Ginibre's real ensemble [4]. 
Theorem 2.3. As $N \rightarrow \infty, s_{1}^{(N)}$ converges weakly in the sense of measures to the uniform law on $[-\sqrt{2}, \sqrt{2}]$, and $s_{2}^{(N)}$ converges in the same manner to the semi-circular law with the same support. In particular, it is proved that

$$
\int e^{i t x} s_{1}^{(N)}(x) d x \rightarrow \frac{1}{\sqrt{2} t} \sin (\sqrt{2} t)
$$

and

$$
\int e^{i t x} s_{2}^{(N)}(x) d x \rightarrow \frac{\sqrt{2}}{t} J_{1}(\sqrt{2} t),
$$

where the convergence is pointwise.

We give an elementary proof of the above, making use of the explicit skew-orthogonal polynomial system derived below. Given that the number of charge 1 particles is $o(N)$, one could undoubtedly make a large deviation proof along the lines of [2] or [3] of a stronger version of the second statement: that the random counting measure of charge 2 particles converges almost surely to the semi-circle law. However, it is not clear how to use such energy optimization ideas to access the charge 1 profile.
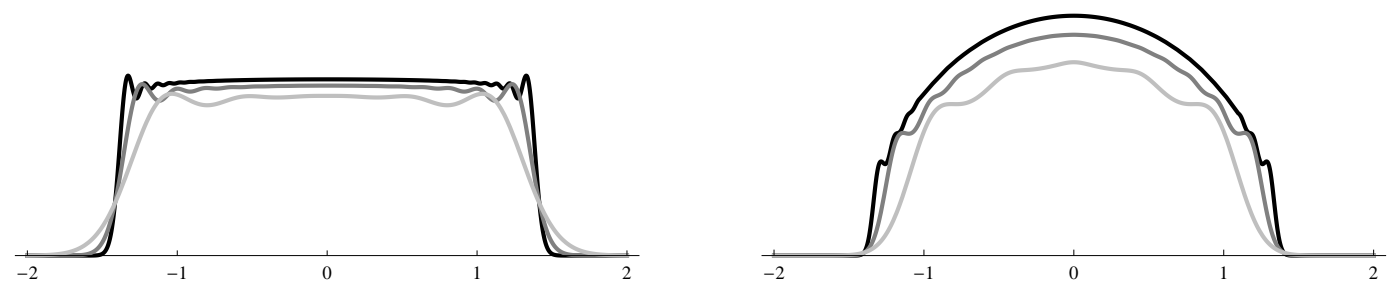

Figure 1: $s_{1}^{(N)}$ (left) and $s_{2}^{(N)}$ (right) for, from lightest to darkest, $N=10,30$ and 90.

\section{A Pfaffian point process for the particles}

All of the results in this paper follow, in one way or another, from the fact that our interacting particles form a Pfaffian point process very much like that of Ginibre's real ensemble and related to the Gaussian Orthogonal and Symplectic Ensembles.

The results in this section are valid for quite general weight functions $w$ and fugacities. Thus, for the time being, we will return to the general situation.

\subsection{The joint density of particles}

The joint density of particles for a particular choice of $(L, M)$ is given by

$$
\frac{X^{L}}{Z(X)} \Omega_{L, M}(\boldsymbol{\alpha}, \boldsymbol{\beta}), \quad \text { where } \quad \Omega_{L, M}(\boldsymbol{\alpha}, \boldsymbol{\beta})=e^{-E(\boldsymbol{\alpha}, \boldsymbol{\beta})} .
$$

More specifically,

$$
\Omega_{L, M}(\boldsymbol{\alpha}, \boldsymbol{\beta})=\prod_{\ell=1}^{L} w\left(\alpha_{\ell}\right) \prod_{m=1}^{M} w\left(\beta_{m}\right)^{2} \prod_{j<k}\left|\alpha_{j}-\alpha_{k}\right| \prod_{m<n}\left|\beta_{m}-\beta_{n}\right|^{4} \prod_{\ell=1}^{L} \prod_{m=1}^{M}\left|\alpha_{\ell}-\beta_{m}\right|^{2} ;
$$

where, for now, the only assumptions we will make on $w$ are that it is positive and Lebesgue measurable with $0<Z(X)<\infty$. 


\subsection{Correlation Functions}

Given $0 \leq \ell \leq L$ and $0 \leq m \leq M$, we define the $\ell, m$-correlation function $R_{\ell, m}^{(N)}: \mathbb{R}^{\ell} \times \mathbb{R}^{m} \rightarrow$ $[0, \infty)$ by

$$
R_{\ell, m}^{(N)}(\mathbf{x} ; \mathbf{y})=\sum_{\substack{(L, M) \\ L \geq \ell, M \geq m}} \frac{1}{(L-\ell) !(M-m) !} \int_{\mathbb{R}^{L-\ell}} \int_{\mathbb{R}^{M-m}} \Omega_{L, M}(\mathbf{x} \vee \boldsymbol{\alpha}, \mathbf{y} \vee \boldsymbol{\beta}) d \mu^{L-\ell}(\boldsymbol{\alpha}) d \mu^{M-m}(\boldsymbol{\beta}),
$$

where, for instance, $\mathbf{x} \vee \boldsymbol{\alpha}$ is the vector in $\mathbb{R}^{L}$ formed by concatenating $\mathbf{x} \in \mathbb{R}^{\ell}$ and $\boldsymbol{\alpha} \in \mathbb{R}^{L-\ell}$. We will often write $R_{\ell, m}$ for $R_{\ell, m}^{(N)}$ in situations where $N$ is seen as being fixed.

The correlation functions encode statistical information about the configurations of the charged particles. To be more precise, given $\boldsymbol{\alpha} \in \mathbb{R}^{L}$ and $\boldsymbol{\beta} \in \mathbb{R}^{M}$ with $L+2 M=N$, we set

$$
\xi=\xi(\boldsymbol{\alpha}, \boldsymbol{\beta})=\left(\xi_{1}, \xi_{2}\right)=\left(\xi_{1}(\boldsymbol{\alpha}), \xi_{2}(\boldsymbol{\beta})\right)=\left(\left\{\alpha_{1}, \ldots, \alpha_{\ell}\right\},\left\{\beta_{1}, \ldots \beta_{m}\right\}\right) .
$$

Given an $L$-tuple of mutually disjoint subsets of $\mathbb{R}, \mathbf{A}=\left(A_{1}, A_{2}, \ldots, A_{L}\right)$, and an $M$-tuple of mutually disjoint subsets of $\mathbb{R}, \mathbf{B}=\left(B_{1}, B_{2}, \ldots, B_{M}\right)$, the probability that the system is in a state where there is exactly one charge 1 particle in each of the $A_{\ell}$ and exactly one charge 2 particle in each of the $B_{m}$ is given by

$$
\begin{aligned}
\operatorname{Prob}\left\{\left|A_{1} \cap \xi_{1}\right|=1, \ldots,\left|A_{L} \cap \xi_{1}\right|=1,\left|B_{1} \cap \xi_{2}\right|=1, \ldots,\left|B_{M} \cap \xi_{2}\right|=1\right\} \\
=\mathbb{E}\left[\left\{\prod_{\ell=1}^{L}\left|A_{\ell} \cap \xi_{1}\right|\right\}\left\{\prod_{m=1}^{M}\left|B_{m} \cap \xi_{2}\right|\right\}\right] .
\end{aligned}
$$

This probability can also be represented by

$$
\frac{1}{L ! M !} \sum_{\sigma \in S_{L}} \sum_{\tau \in S_{M}} \int_{B_{\tau(1)}} \cdots \int_{B_{\tau(M)}} \int_{A_{\sigma(1)}} \cdots \int_{A_{\sigma}(L)} \Omega_{L, M}(\boldsymbol{\alpha}, \boldsymbol{\beta}) d \mu^{L}(\boldsymbol{\alpha}) d \mu^{M}(\boldsymbol{\beta}) .
$$

Since the integrand is symmetric in the coordinates of $\boldsymbol{\alpha}$ and $\boldsymbol{\beta}$, we find

$$
\mathbb{E}\left[\left\{\prod_{\ell=1}^{L}\left|A_{\ell} \cap \xi_{1}\right|\right\}\left\{\prod_{m=1}^{M}\left|B_{m} \cap \xi_{2}\right|\right\}\right]=\int_{\mathbf{B}} \int_{\mathbf{A}} \Omega_{L, M}(\boldsymbol{\alpha}, \boldsymbol{\beta}) d \mu^{L}(\boldsymbol{\alpha}) d \mu^{M}(\boldsymbol{\beta}) .
$$

The correlation functions can be used to generalize this formula. If $\mathbf{A}=\left(A_{1}, A_{2}, \ldots, A_{\ell}\right)$ is a tuple of disjoint subsets of $\mathbb{R}$ and $\mathbf{B}=\left(B_{1}, B_{2}, \ldots, B_{m}\right)$ another such tuple, then

$$
\mathbb{E}\left[\left\{\prod_{j=1}^{\ell}\left|A_{j} \cap \xi_{1}\right|\right\}\left\{\prod_{k=1}^{m}\left|B_{k} \cap \xi_{2}\right|\right\}\right]=\int_{\mathbf{B}} \int_{\mathbf{A}} R_{\ell, m}(\mathbf{x} ; \mathbf{y}) d \mu^{\ell}(\boldsymbol{\alpha}) d \mu^{m}(\boldsymbol{\beta}) .
$$

\subsection{Pfaffian point processes}

Consider, for the moment, a simplified system of indistinguishable random points $\zeta=$ $\left\{\gamma_{1}, \gamma_{2}, \ldots, \gamma_{N}\right\} \subseteq \mathbb{R}$ with correlation functions $R_{n}(\mathbf{z})$ satisfying

$$
\mathbb{E}\left[\prod_{j=1}^{n}\left|A_{j} \cap \zeta\right|\right]=\int_{A_{1}} \cdots \int_{A_{n}} R_{n}(\mathbf{z}) d \mu^{n}(\mathbf{z})
$$


for any $n$-tuple $\left(A_{1}, A_{2}, \ldots, A_{n}\right)$ of mutually disjoint sets.

If there exists a matrix valued function $K_{N}: \mathbb{R}^{2} \rightarrow \mathbb{R}^{2 \times 2}$ such that

$$
R_{n}(\mathbf{z})=\operatorname{Pf}\left[K_{N}\left(z_{j}, z_{k}\right)\right]_{j, k=1}^{n},
$$

then we say that our ensemble of random points forms a Pfaffian point process with matrix kernel $K_{N}$. Much of the information about probabilities of locations of particles (e.g. gap probabilities) can be derived from properties of the matrix kernel. Moreover, in many instances, we are interested in statistical properties of the particles as their number (or some related parameter) tends toward $\infty$. In these instances, it is sometimes possible to analyze $K_{N}(x, y)$ in this limit (under, perhaps, some scaling of $x$ and $y$ dependent on $N$ ) so that the relevant limiting probabilities are attainable from this limiting kernel.

For the ensemble of charge 1 and charge 2 particles with total charge $N$, we will demonstrate that the correlation functions have a Pfaffian formulation of the form,

$$
R_{\ell, m}(\mathbf{x} ; \mathbf{y})=2^{\ell} \operatorname{Pf}\left[\begin{array}{ll}
K_{N}^{1,1}\left(x_{j}, x_{j^{\prime}}\right) & K_{N}^{1,2}\left(x_{j}, z_{k^{\prime}}\right) \\
K_{N}^{2,1}\left(z_{k}, x_{k^{\prime}}\right) & K_{N}^{2,2}\left(x_{k}, x_{k^{\prime}}\right)
\end{array}\right] ; \quad \begin{aligned}
& j, j^{\prime}=1,2, \ldots, \ell \\
& k, k^{\prime}=1,2, \ldots m
\end{aligned}
$$

where $K_{N}^{1,1}, K_{N}^{1,2}, K_{N}^{2,1}$ and $K_{N}^{2,2}$ are $2 \times 2$ matrix kernels.

\subsection{A Pfaffian form for the total partition function}

In order to establish the existence of the matrix kernels we first need a Pfaffian formulation of the total partition function.

Given a measure $\nu$ on $\mathbb{R}$ we define the operators $\epsilon_{1}^{\nu}$ and $\epsilon_{2}^{\nu}$ on $L^{2}(\nu)$ by

$$
\epsilon_{1}^{\nu} f(x)=\frac{1}{2} \int_{\mathbb{R}} f(y) \operatorname{sgn}(y-x) d \nu(y) \quad \text { and } \quad \epsilon_{2}^{\nu} f(y)=f^{\prime}(y) .
$$

(Obviously $\epsilon_{2}^{\nu}$ does not depend on $\nu$, but it is convenient to maintain symmetric notation). Using these inner products we define

$$
\langle f \mid g\rangle_{b^{2}}^{\nu}=\int_{\mathbb{R}}\left[f(x) \epsilon_{b} g(x)-g(x) \epsilon_{b} f(x)\right] d \nu(x), \quad b=1,2 .
$$

We specialize these operators and inner products for Lebesgue measure $\mu$ by setting $\epsilon_{b}=\epsilon_{b}^{\mu}$ and $\langle f \mid g\rangle_{b^{2}}^{\mu}$. We also write $\widetilde{f}(x)=w(x) f(x)$. It is easily seen that

$$
\langle\widetilde{f} \mid \widetilde{g}\rangle_{1}=\int_{\mathbb{R}}\left[\widetilde{f}(x) \epsilon_{1} \widetilde{g}(x)-\widetilde{g}(x) \epsilon_{1} \widetilde{f}(x)\right] d \mu(x)=\langle f \mid g\rangle_{1}^{w \mu} .
$$

Similarly,

$$
\begin{aligned}
\langle\widetilde{f} \mid \widetilde{g}\rangle_{4} & =\int_{\mathbb{R}}\left[\widetilde{f}(x) \frac{d}{d x} \widetilde{g}(x)-\widetilde{g}(x) \frac{d}{d x} \widetilde{f}(x)\right] d \mu(x) \\
& =\int_{\mathbb{R}} w(x)^{2}\left[f(x) g^{\prime}(x)-g(x) f^{\prime}(x)\right] d x=\langle f \mid g\rangle_{4}^{w^{2} \mu} .
\end{aligned}
$$

We call a family of polynomials, $\mathbf{p}=\left(p_{0}(x), p_{1}(x), \ldots, p_{N-1}(x)\right)$, a complete family of polynomials if $\operatorname{deg} p_{n}=n$. A complete family of monic polynomials is defined accordingly. 
Theorem 3.1. Suppose $N$ is even and $\mathbf{p}$ is any complete family of monic polynomials. Then,

$$
Z(X)=\operatorname{Pf}\left(X^{2} \mathbf{A}^{\mathbf{p}}+\mathbf{B}^{\mathbf{p}}\right)
$$

where

$$
\mathbf{A}^{\mathbf{p}}=\left[\left\langle\widetilde{p}_{m} \mid \widetilde{p}_{n}\right\rangle_{1}\right]_{m, n=0}^{N-1} \quad \text { and } \quad \mathbf{B}^{\mathbf{p}}=\left[\left\langle\widetilde{p}_{m} \mid \widetilde{p}_{n}\right\rangle_{4}\right]_{m, n=0}^{N-1}
$$

Corollary 3.2. With the same assumptions as Theorem [3.1, $Z=\operatorname{Pf}\left(\mathbf{A}^{\mathbf{p}}+\mathbf{B}^{\mathbf{p}}\right)$.

\subsection{A Pfaffian formulation of the correlation functions}

In order to describe the entries in the kernels $K_{N}^{1,1}, K_{N}^{1,2}, K_{N}^{2,1}$ and $K_{N}^{2,2}$, we suppose $\mathbf{p}$ is any complete family of polynomials and define

$$
\mathbf{C}^{\mathbf{p}}=\mathbf{A}^{\mathbf{p}}+\mathbf{B}^{\mathbf{p}}
$$

where $\mathbf{A}^{\mathbf{p}}$ and $\mathbf{B}^{\mathbf{p}}$ are as in Corollary 3.2. (Here we are setting $X=1$, though similar maneuvers are valid for general $X>0$ ). Since we are assuming that $Z=\operatorname{Pf} \mathbf{C}^{\mathbf{p}}$ is non-zero, $\mathbf{C}^{\mathbf{p}}$ is invertible and we set

$$
\left(\mathbf{C}^{\mathbf{p}}\right)^{-\mathbf{T}}=\left[\zeta_{j, k}\right]_{j, k=0}^{N-1}
$$

The $\zeta_{j, k}$ clearly depend on our choice of polynomials. We then define

$$
\varkappa_{N}(x, y)=\sum_{j, k=0}^{N-1} \widetilde{p}_{j}(x) \zeta_{j, k} \widetilde{p}_{k}(y) .
$$

The operators $\epsilon_{1}$ and $\epsilon_{2}$ operate on $\varkappa_{N}(x, y)$ in the usual manner. For instance,

$$
\epsilon_{2} \varkappa_{N}(x, y)=\sum_{j, k=0}^{N-1} \epsilon_{2} \widetilde{p}_{j}(x) \zeta_{j, k} \epsilon_{2} \widetilde{p}_{k}(y)
$$

and

$$
\varkappa_{N} \epsilon_{1}(x, y)=\sum_{j, k=0}^{N-1} \widetilde{p}_{j}(x) \zeta_{j, k} \epsilon_{1} \widetilde{p}_{k}(y) .
$$

(That is, $\epsilon$ written on the left acts on the $\varkappa_{N}(x, y)$ viewed as a function of $x$, etc.).

Theorem 3.3. Suppose $N$ is even, $\mathbf{p}$ is any complete family of polynomials and $\varkappa_{N}(x, y)$ is given as in (3.1). Then,

$$
R_{\ell, m}(\mathbf{x} ; \mathbf{y})=2^{\ell} \operatorname{Pf}\left[\begin{array}{ll}
K_{N}^{1,1}\left(x_{j}, x_{j^{\prime}}\right) & K_{N}^{1,2}\left(x_{j}, z_{k^{\prime}}\right) \\
K_{N}^{2,1}\left(z_{k}, x_{k^{\prime}}\right) & K_{N}^{2,2}\left(x_{k}, x_{k^{\prime}}\right)
\end{array}\right] ; \quad \begin{aligned}
& j, j^{\prime}=1,2, \ldots, \ell \\
& k, k^{\prime}=1,2, \ldots m
\end{aligned}
$$

where

$$
\begin{aligned}
& K_{N}^{1,1}(x, y)=\left[\begin{array}{cc}
\varkappa_{N}(x, y) & \varkappa_{N} \epsilon_{1}(x, y) \\
\epsilon_{1} \varkappa_{N}(x, y) & \epsilon_{1} \varkappa_{N} \epsilon_{1}(x, y)+\frac{1}{4} \operatorname{sgn}(y-x)
\end{array}\right], \\
& K_{N}^{2,2}(x, y)=\left[\begin{array}{cc}
\varkappa_{N}(x, y) & \varkappa_{N} \epsilon_{2}(x, y) \\
\epsilon_{2} \varkappa_{N}(x, y) & \epsilon_{2} \varkappa_{N} \epsilon_{2}(x, y)
\end{array}\right] \\
& K_{N}^{1,2}(x, y)=\left[\begin{array}{cc}
\varkappa_{N}(x, y) & \varkappa_{N} \epsilon_{1}(x, y) \\
\epsilon_{2} \varkappa_{N}(x, y) & \epsilon_{2} \varkappa_{N} \epsilon_{1}(x, y)
\end{array}\right] \quad \text { and } \quad K_{N}^{2,1}(x, y)=\left[\begin{array}{cc}
\varkappa_{N}(x, y) & \varkappa_{N} \epsilon_{2}(x, y) \\
\epsilon_{1} \varkappa_{N}(x, y) & \epsilon_{1} \varkappa_{N} \epsilon_{2}(x, y)
\end{array}\right] \text {. }
\end{aligned}
$$


Remark. The factor $2^{\ell}$ can be moved inside the Pfaffian so that the entries in the various kernels where an $\epsilon_{1}$ appears are multiplied by 2 . This maneuver is superficial, but has the effect of making these particular entries appear more like the entries in other $\beta=1$ ensembles (e.g. GOE). For instance, $\frac{1}{2} \operatorname{sgn}(y-x)$ appears more natural to experts used to these other ensembles.

We can simplify the presentation of the matrix kernels with a bit of notation. First, let us write

$$
K_{N}(x, y)=\left[\begin{array}{ll}
\varkappa_{N}(x, y) & \varkappa_{N}(x, y) \\
\varkappa_{N}(x, y) & \varkappa_{N}(x, y)
\end{array}\right], \quad \text { and } \quad E_{b}=\left[\begin{array}{cc}
1 & 0 \\
0 & \epsilon_{b}
\end{array}\right] ; \quad b=1,2 .
$$

Then,

$$
\begin{gathered}
K_{N}^{1,1}(x, y)=E_{1} K_{N}(x, y) E_{1}+\left[\begin{array}{cc}
0 & 0 \\
0 & \frac{1}{4} \operatorname{sgn}(y-x)
\end{array}\right], \\
K_{N}^{2,2}(x, y)=E_{2} K_{N}(x, y) E_{2}, \quad K_{N}^{1,2}(x, y)=E_{1} K_{N}(x, y) E_{2}, \quad K_{N}^{2,1}(x, y)=E_{2} K_{N}(x, y) E_{1} .
\end{gathered}
$$
by

We notice in particular that the functions $R_{1,0}^{(N)}$ and $R_{0,1}^{(N)}$ given in Section 2.2 are given

$$
R_{1,0}^{(N)}(x)=2 \sum_{j, k=0}^{N-1} \widetilde{p}_{j}(x) \zeta_{j, k} \epsilon_{1} \widetilde{p}_{k}(x) \quad \text { and } \quad R_{0,1}^{(N)}(x)=\sum_{j, k=0}^{N-1} \widetilde{p}_{j}(x) \zeta_{j, k} \epsilon_{2} \widetilde{p}_{k}(x) .
$$

\subsection{Skew-orthogonal polynomials}

The entries in the kernel themselves can be simplified (or at least presented in a simplified form) by a judicious choice of $\mathbf{p}$. If we define

$$
\langle f \mid g\rangle=\langle f \mid g\rangle_{1}+\langle f \mid g\rangle_{4},
$$

then

$$
\mathbf{C}^{\mathbf{p}}=\left[\left\langle\widetilde{p}_{m} \mid \widetilde{p}_{n}\right\rangle\right]_{m, n=0}^{N-1} .
$$

Since $\varkappa_{N}$ (and by extension all other entries of the various kernels) depend on the inverse transpose of $\mathbf{C}^{\mathbf{p}}$, it is desirable to find a complete family of polynomials for which $\mathbf{C}^{\mathbf{p}}$ can be easily inverted.

We say $\mathbf{p}=\left(p_{0}, p_{1}, \ldots\right)$ is a family of skew-orthogonal polynomials for the skew-inner product $\langle\cdot \mid \cdot\rangle$ with weight $w$ if there exists real numbers (called normalizations) $r_{1}, r_{2}, \ldots$ such that

$$
\left\langle\widetilde{p}_{2 j} \mid \widetilde{p}_{2 k}\right\rangle=\left\langle\widetilde{p}_{2 j+1} \mid \widetilde{p}_{2 k+1}\right\rangle=0 \quad \text { and } \quad\left\langle\widetilde{p}_{2 j} \mid \widetilde{p}_{2 k+1}\right\rangle=-\left\langle\widetilde{p}_{2 k+1} \mid \widetilde{p}_{2 j}\right\rangle=\delta_{j, k} r_{j} .
$$

Using these polynomials, the entries in the matrix kernels presented in Section 3.5 have a particularly simple form. For instance,

$$
\varkappa_{N}(x, y)=\sum_{j=0}^{J-1} \frac{\widetilde{p}_{2 j}(x) \widetilde{p}_{2 j+1}(y)-\widetilde{p}_{2 j+1}(x) \widetilde{p}_{2 j}(y)}{r_{j}},
$$

and the entries of the kernels are computed by applying the appropriate $\epsilon$ operators to this expression. 


\subsection{Specification to the Harmonic Oscillator Potential}

We now return to the case where the weight function is $w(x)=e^{-x^{2} / 2}$.

Theorem 3.4. Let

$$
\langle\cdot \mid \cdot\rangle^{(X)}=X^{2}\langle\cdot \mid \cdot\rangle_{1}+\langle\cdot \mid \cdot\rangle_{4} .
$$

A complete family of skew-orthogonal polynomials for the weight $w$ with respect to $\langle\cdot \mid \cdot\rangle(X)$ is given by

$$
P_{2 j}^{(X)}(x)=\sum_{k=0}^{j}(-1)^{k} \frac{L_{k}\left(-X^{2}\right)}{L_{k}(0)} L_{k}\left(x^{2}\right),
$$

and

$$
\begin{aligned}
P_{2 j+1}^{(X)}(x) & =2 x P_{2 j}^{(X)}(x)-2 \frac{d}{d x} P_{2 j}^{(X)}(x) \\
& =4 X^{2} x \sum_{k=0}^{m-1}(-1)^{k} \frac{L_{k}^{\frac{1}{2}}\left(-X^{2}\right)}{L_{k}^{\frac{1}{2}}(0)} L_{k}^{\frac{1}{2}}\left(x^{2}\right)+2 x(-1)^{m} \frac{L_{m}^{-\frac{1}{2}}\left(-X^{2}\right)}{L_{m}^{-\frac{1}{2}}(0)} L_{m}^{\frac{1}{2}}\left(x^{2}\right) .
\end{aligned}
$$

where $L_{k}(x)=L_{k}^{(-1 / 2)}(x)$ is the generalized kth Laguerre polynomial. The normalization of this family of polynomials is given by

$$
\left\langle\widetilde{P}_{2 m}^{(X)} \mid \widetilde{P}_{2 m+1}^{(X)}\right\rangle^{(X)}=\frac{4 \pi(m+1) !}{\Gamma\left(m+\frac{1}{2}\right)} L_{m}\left(-X^{2}\right) L_{m+1}\left(-X^{2}\right) .
$$

We can recover a family of monic skew-orthogonal polynomials by dividing by the leading coefficient. Specifically,

Corollary 3.5. A complete family of monic skew-orthogonal polynomials for the weight $w$ with respect to $\langle\cdot \mid \cdot\rangle^{(X)}$ is given by

$$
p_{2 j}^{(X)}(x)=\frac{L_{j}(0) j !}{L_{j}\left(-X^{2}\right)} \sum_{k=0}^{j}(-1)^{k} \frac{L_{k}\left(-X^{2}\right)}{L_{k}(0)} L_{k}\left(x^{2}\right),
$$

and

$$
p_{2 j+1}^{(X)}(x)=x p_{2 j}^{(X)}(x)-\frac{d}{d x} p_{2 j}^{(X)}(x) .
$$

The normalization for this family of monic skew-orthogonal polynomials is given by

$$
r_{j}^{(X)}=\left\langle\widetilde{p}_{2 j}^{(X)} \mid \widetilde{p}_{2 j+1}^{(X)}\right\rangle^{(X)}=4 \frac{(j+1) ! \Gamma\left(j+\frac{1}{2}\right)}{j !} \frac{L_{j+1}\left(-X^{2}\right)}{L_{j}\left(-X^{2}\right)} .
$$

Setting $X=1$, we recover a family of skew-orthogonal polynomials for the harmonic oscillator two charge ensemble with fugacity equal to one, and we will write $p_{n}$ for $p_{n}^{(1)}$ and $r_{j}$ for $r_{j}^{(1)}$. 


\section{Proofs}

\subsection{Proof of Theorem 2.1}

We set $J=N / 2$. To prove 1 , we use Theorem 3.1 and the skew-orthogonal polynomials from Corollary 3.5 to write

$$
Z(X)=\operatorname{Pf}\left[\begin{array}{ccccc}
0 & r_{0}^{(X)} & & & \\
-r_{0}^{(X)} & 0 & & & \\
& & \ddots & & \\
& & & 0 & r_{J-1}^{(X)} \\
& & & -r_{J-1}^{(X)} & 0
\end{array}\right]=\prod_{j=0}^{J-1} r_{j}^{(X)} .
$$

Hence,

$$
\frac{Z(X)}{Z}=\prod_{j=0}^{J-1} \frac{r_{j}^{(X)}}{r_{j}}=\frac{L_{J}\left(-X^{2}\right) L_{0}(-1)}{L_{J}(-1) L_{0}\left(-X^{2}\right)}=\frac{L_{J}\left(-X^{2}\right)}{L_{J}(-1)}
$$

where again $L_{J}(x)=L_{J}^{(-1 / 2)}(x)$. Note $L_{0}(X)=1$.

The remaining claims follow from the above by definition and the properties of Laguerre polynomials.

\subsection{Proof of Theorem 2.2}

Point 3 of Theorem 2.1 specified to the first two moments produces

$$
\mathbb{E}[L]=\frac{d}{d X}\left[\frac{Z(X)}{Z}\right]_{X=1}, \quad \operatorname{Var}(L)=\left[\frac{d}{d X}\left(X \frac{d}{d X} \frac{Z(X)}{Z}\right)-\left(\frac{d}{d X} \frac{Z(X)}{Z}\right)^{2}\right]_{X=1} .
$$

Now, since $L_{J}^{\prime}(x)=-L_{J-1}^{1 / 2}(x)$ and $L_{J}(x)=L_{J}^{1 / 2}(x)-L_{J-1}^{1 / 2}(x)$, we have that

$$
\mathbb{E}(L)=2 \frac{L_{J-1}^{1 / 2}(-1)}{L_{J}(-1)}=2 \frac{L_{J}^{1 / 2}(-1)}{L_{J}(-1)}-2 .
$$

Further, using the differential equation $x L_{J}^{\prime \prime}(x)+(1 / 2-x) L_{J}^{\prime}(x)+J L_{J}(x)=0$, we also have that

$$
\begin{aligned}
\frac{d}{d x}\left(x \frac{d}{d x} L_{J}\left(-x^{2}\right)\right) & =-4 x L_{J}^{\prime}\left(-x^{2}\right)+4 x^{3} L_{J}^{\prime \prime}\left(-x^{2}\right) \\
& =\left(-2 x+4 x^{3}\right) L_{J}^{\prime}\left(-x^{2}\right)+4 x J L_{J}\left(-x^{2}\right) .
\end{aligned}
$$

This yields

$$
\operatorname{Var}(L)=4 J-\mathbb{E}(L)-\mathbb{E}(L)^{2},
$$

and so asymptotics of the variance follow from those for the mean.

Next introduce a version of Perron's formula (see [5]),

$$
L_{n}^{\alpha}(-1)=\frac{1}{2 \sqrt{\pi e}} m^{\alpha / 2-1 / 4} e^{2 \sqrt{m}}\left(1+C_{1}(\alpha) m^{-1 / 2}+C_{2}(\alpha) m^{-1}+\mathrm{O}\left(m^{-3 / 2}\right)\right),
$$


where $m=n+1$ and $C_{j}(\alpha)$ are known explicitly. In particular, $C_{1}(1 / 2)=-1 / 6, C_{2}(1 / 2)=$ $-7 / 144, C_{1}(-1 / 2)=-2 / 3$, and $C_{2}(-1 / 2)=77 / 144$. Substituting into the above we then obtain

$$
\mathbb{E}(L)=2 \sqrt{J+1}-1-\frac{2}{3 \sqrt{J+1}}+\mathrm{O}\left(J^{-1}\right)=2 \sqrt{J}-1+\frac{1}{3 \sqrt{J}}+\mathrm{O}\left(J^{-1}\right),
$$

and $\operatorname{Var}(L)=2 \sqrt{J}-\frac{4}{3}+O\left(J^{-1 / 2}\right)$ which completes the proof of point 1 (recall $J=N / 2$ ).

Moving to the limit law for $L$, we introduce a little new notation. Set

$$
p_{N}(k)=\frac{C_{N}}{\Gamma\left(\frac{N}{2}-\frac{k}{2}+1\right) \Gamma\left(\frac{k}{2}+\frac{1}{2}\right) \Gamma\left(\frac{k}{2}+1\right)}=C_{N} q_{N}(k)^{-1}
$$

with $C_{N}=\Gamma\left(\frac{N}{2}+\frac{1}{2}\right)\left[L_{N / 2}(-1)\right]^{-1}$. For $k$ even, $p_{N}(k)$ is the probability of $k$ particles of charge 1, otherwise this probability is zero, compare point 1 of Theorem 2.1. In the continuum limit this distinction is unimportant; we will show that, as $N \rightarrow \infty$

$$
(2 N)^{1 / 4} p_{N}\left((2 N)^{1 / 2}+(2 N)^{1 / 4} c\right)=\frac{e^{-c^{2} / 2}}{\sqrt{2 \pi}}\left(1+O\left(N^{-1 / 4}\right)\right)
$$

uniformly for $c$ on compact sets.

First note that by Stirling's approximation (in the form $\Gamma(z)=\sqrt{\frac{2 \pi}{z}}(z / e)^{z}\left(1+O\left(\frac{1}{z}\right)\right)$ ) and again Perron's formula (now in the simpler form $L_{z}(-1)=\frac{1}{2 \sqrt{\pi e z}} e^{2 \sqrt{z}}\left(1+O\left(\frac{1}{\sqrt{z}}\right)\right)$ ),

$$
C_{N}=2 \pi \sqrt{N e}(N / 2)^{(N / 2)} e^{-N / 2-\sqrt{2 N}}\left(1+O\left(N^{-1 / 2}\right)\right) .
$$

Next, with both $k$ and $N-k$ large we have

$$
\begin{aligned}
q_{N}(k)= & (2 \pi)^{3 / 2} \sqrt{N k}(N / 2)^{(N / 2)} e^{-N / 2-k / 2} \\
& \times e^{\left[(N / 2-k / 2) \log (1-k / N)+(k / 2) \log \left(k^{2} / 2 N\right)\right]}\left(1+O\left(k^{-1} \vee(N-k)^{-1} \vee k N^{-1}\right)\right),
\end{aligned}
$$

again by Stirling's approximation. Restricting to $k=O(\sqrt{N})$, (4.2) and (4.3) yield

$$
p_{N}(k)=\sqrt{\frac{e}{2 \pi k}} e^{-\phi_{N}(k)}\left(1+O\left(N^{-1 / 2}\right)\right),
$$

where

$$
\phi_{N}(k)=\sqrt{2 N}-\frac{k}{2}+\left(\frac{N}{2}-\frac{k}{2}\right) \log \left(1-\frac{k}{N}\right)+\frac{k}{2} \log \left(\frac{k^{2}}{2 N}\right) .
$$

Now, quite simply

$$
\left(\frac{N}{2}-\frac{k}{2}\right) \log \left(1-\frac{k}{N}\right)=-\frac{k}{2}+\frac{k^{2}}{4 N}+O\left(N^{-1 / 2}\right),
$$

if $k=O(\sqrt{N})$, and, if $k$ is also such that $1-\frac{k^{2}}{2 N}=O\left(N^{-1 / 4}\right)$, we further have

$$
\frac{k}{2} \log \left(\frac{k^{2}}{2 N}\right)=-\frac{k}{2}\left(1-\frac{k^{2}}{2 N}\right)-\frac{k}{4}\left(1-\frac{k^{2}}{2 N}\right)^{2}+O\left(N^{-1 / 4}\right) .
$$

More precisely, from the last two displays we readily find that

$$
\phi_{N}(\sqrt{2 N}+\ell)=\frac{1}{2}+\frac{\ell^{2}}{2 \sqrt{2 N}}+O\left(N^{-1 / 4}\right), \text { uniformly for } \ell=O\left(N^{1 / 4}\right) .
$$


Substituting back into (4.4), since $(\sqrt{2 N}+\ell)^{-1 / 2}=(2 N)^{-1 / 4}\left(1+O\left(N^{-1 / 4}\right)\right)$ again for $\ell=O\left(N^{-1 / 4}\right)$, completes the verification of (4.1).

Last, for the tail estimate, revisiting (4.2) and (4.3) shows the conclusion of (4.4) may be modified to read

$$
C^{-1} k^{-1 / 2} e^{-\phi_{N}(k)} \leq p_{N}(k) \leq C e^{-\phi_{N}(k)},
$$

for all $1 \leq k \leq N$ with a numerical constant $C$. (Here we understand $\left(1-\frac{k}{N}\right) \log \left(1-\frac{k}{N}\right)$ to be zero at $k=N$.) Differentiating yields

$$
\frac{d}{d k} \phi_{N}(k)=\frac{1}{2} \log \left(\frac{k^{2}}{2(N-k)}\right),
$$

and so $\phi_{N}(k)$ is decreasing for $k<c^{-1} \sqrt{2 n}$ and increasing for $k>c \sqrt{2 N}$ for any $c>1$. Now, since $(1-\epsilon) \log (1-\epsilon) \geq-\epsilon$ and $\log (1+\epsilon) \geq \epsilon-\epsilon^{2} / 2$ for $0<\epsilon \leq 1$,

$$
\phi_{N}((1+\epsilon) \sqrt{2 N}) \geq-\epsilon \sqrt{2 N}+2(1+\epsilon) \sqrt{2 N} \log (1+\epsilon) \geq \epsilon \sqrt{2 N},
$$

also for $0<\epsilon \leq 1$. Hence, for $c>1, \operatorname{Prob}(L>c \sqrt{2 N}) \leq N p_{N}(c \sqrt{2 N}) \leq$ $C N e^{-((c-1) \wedge 1) \sqrt{2 N}}$. The proof for the left tail is much the same.

\subsection{Proof of Theorem 2.3}

In both cases we use the expression of the one point function in terms of Hermite polynomials, see (4.17) and (4.20) below.

We start with

$$
s_{N}^{(1)}(x)=\sqrt{2} \sum_{n=0}^{N / 2-1} \frac{\epsilon_{1} \widetilde{p}_{2 n+1}(x) \widetilde{p}_{2 n}(\sqrt{N} x)-\widetilde{p}_{2 n+1}(\sqrt{N} x) \epsilon_{1} \widetilde{p}_{2 n}(\sqrt{N} x)}{r_{n}},
$$

and

$$
s_{N}^{(2)}(x)=\frac{2}{\sqrt{N}} \sum_{n=0}^{N / 2-1} \frac{\widetilde{p}_{2 n+1}^{\prime}(\sqrt{N} x) \widetilde{p}_{2 n}(\sqrt{N} x)-\widetilde{p}_{2 n+1}(\sqrt{N} x) \widetilde{p}_{2 n}^{\prime}(\sqrt{N} x)}{r_{n}},
$$

along with the relations $\int_{-\infty}^{x} \widetilde{p}_{2 n+1}=\epsilon_{1} \widetilde{p}_{2 n+1}(x)=2 \widetilde{p}_{2 n}(x)$ and $\widetilde{p}_{2 n}(x)=\epsilon_{2} \widetilde{p}_{2 n}(x)=$ $-\frac{1}{2} \widetilde{p}_{2 n+1}(x)$. An integration by parts in both instances then allows: with $t_{N}=t / \sqrt{N}$,

$$
\begin{aligned}
& \int_{-\infty}^{\infty} e^{i t x} s_{N}^{(1)}(x) d x \\
& =\frac{4 \sqrt{2}}{\sqrt{N}} \sum_{n=0}^{N / 2-1} r_{n}^{-1} \int_{-\infty}^{\infty} e^{i t_{N} x}\left(\widetilde{p}_{2 n}(x)\right)^{2} d x-\frac{2 \sqrt{2} i t_{N}}{\sqrt{N}} \sum_{n=0}^{N / 2-1} r_{n}^{-1} \int_{-\infty}^{\infty} e^{i t_{N} x} \widetilde{p}_{2 n}(x) \epsilon_{1} \widetilde{p}_{2 n}(x) d x,
\end{aligned}
$$

and

$$
\begin{aligned}
& \int_{-\infty}^{\infty} e^{i t x} s_{N}^{(2)}(x) d x \\
& =\frac{2}{N} \sum_{n=0}^{N / 2-1} r_{n}^{-1} \int_{-\infty}^{\infty} e^{i t_{N} x}\left(\widetilde{p}_{2 n+1}(x)\right)^{2} d x-\frac{2 i t_{N}}{N} \sum_{n=0}^{N / 2-1} r_{n}^{-1} \int_{-\infty}^{\infty} e^{i t_{N} x} \widetilde{p}_{2 n}(x) \widetilde{p}_{2 n+1}(x) d x .
\end{aligned}
$$

The first, and primary, step is to show that the advertised limits stem from the first sums on the right of the above expressions. 
Lemma 4.1. Let $\hat{s}_{N}^{(1)}(t)$ and $\hat{s}_{N}^{(2)}(t)$ denote, respectively, the first term on the right hand side of (4.5) and 4.6). Then,

$$
\hat{s}_{N}^{(1)}(t) \rightarrow \frac{\sin \sqrt{2} t}{\sqrt{2} t}, \quad \hat{s}_{N}^{(2)}(t) \rightarrow \frac{\sqrt{2}}{t} J_{1}(\sqrt{2} t)
$$

as $N \rightarrow \infty$.

Proof. Since $\widetilde{H}_{k}(x)=H_{k}(x) e^{-x^{2} / 2}$ are the eigenfunctions of the Fourier transform - in particular $\left(\widetilde{H}_{k}\right)^{\Upsilon}(x)=\frac{1}{\sqrt{2 \pi}} \int_{-\infty}^{\infty} e^{i x u} \widetilde{H}_{k}(u) d u=i^{k} H_{k}(x)$ - we have that

$$
\begin{aligned}
\widehat{\widetilde{p}_{2 n}}(x) & =\sum_{k=0}^{n}(-1)^{k} a_{k} \widetilde{H}_{2 k}(x), \\
\widehat{\widetilde{p}_{2 n+1}}(x) & =i \sum_{k=0}^{n}(-1)^{k} a_{k}\left(\widetilde{H}_{2 k+1}(x)+4 k \widetilde{H}_{2 k-1}(x)\right)=2 i x \sum_{k=0}^{n}(-1)^{k} a_{k} \widetilde{H}_{2 k}(x) .
\end{aligned}
$$

The last equality makes use of the three term recurrence $H_{n+1}(x)=2 x H_{n}(x)-2 n H_{n-1}(x)$. Plancheral's identity then yields,

$$
\hat{s}_{N}^{(1)}(t)=\frac{4 \sqrt{2}}{\sqrt{N}} \sum_{n=0}^{N / 2-1} r_{n}^{-1} \sum_{0 \leq k, \ell \leq n}(-1)^{k+\ell} a_{k} a_{\ell} \int_{-\infty}^{\infty} \widetilde{H}_{2 k}\left(x+t_{N} / 2\right) \widetilde{H}_{2 \ell}\left(x-t_{N} / 2\right) d x,
$$

and

$\hat{s}_{N}^{(2)}(t)=\frac{8}{N} \sum_{n=0}^{N / 2-1} r_{n}^{-1} \sum_{0 \leq k, \ell \leq n}(-1)^{k+\ell} a_{k} a_{\ell} \int_{-\infty}^{\infty}\left(x^{2}-\left(t_{N} / 2\right)^{2}\right) \widetilde{H}_{2 k}\left(x+t_{N} / 2\right) \widetilde{H}_{2 \ell}\left(x-t_{N} / 2\right) d x$.

We begin with the asymptotic considerations of (4.8) which is slightly simpler.

From the expansion $H_{n}(a+b)=\sum_{k=0}^{n}\left(\begin{array}{l}n \\ k\end{array}\right) H_{k}(a)(2 b)^{n-k}$ we find that

$$
\int_{-\infty}^{\infty} \widetilde{H}_{2 k}(x+t / 2) \widetilde{H}_{2 \ell}(x-t / 2) d x=e^{-t^{2} / 4} \sqrt{\pi} \sum_{m=0}^{2(k \wedge \ell)}\left(\begin{array}{c}
2 k \\
m
\end{array}\right)\left(\begin{array}{c}
2 \ell \\
m
\end{array}\right) m !(-2)^{m} t^{2 k+2 \ell-2 m} .
$$

Given this, $\hat{s}_{N}^{(1)}$ is equivalent, as $N \rightarrow \infty$, to

$$
\begin{aligned}
\hat{s}_{N, d}^{(1)} & +\hat{s}_{N, o}^{(1)}=\frac{4 \sqrt{2 \pi}}{\sqrt{N}} \sum_{n=0}^{N / 2-1} r_{n}^{-1} \sum_{k=0}^{n} a_{k}^{2} 2^{2 k}(2 k) ! \sum_{m=0}^{2 k} \frac{(2 k)_{m}}{(m !)^{2}}\left(-\frac{t^{2}}{2 N}\right)^{m} \\
& +\frac{8 \sqrt{2 \pi}}{\sqrt{N}} \sum_{n=1}^{N / 2-1} r_{n}^{-1} \sum_{0 \leq k<\ell \leq n} a_{k} a_{\ell} 2^{k+\ell}(2 k) ! \sum_{m=0}^{2 k} \frac{(2 \ell)_{2 \ell-2 k+m}}{m !(2 \ell-2 k+m) !}\left(-\frac{t^{2}}{2 N}\right)^{\ell-k+m}
\end{aligned}
$$

in which we have introduced a self-evident notation for the diagonal and off-diagonal components as well as the (nontraditional) shorthand $(n)_{m}:=\frac{n !}{(n-m) !}$.

Next, recall the definitions $a_{n}=\frac{n !}{(2 n) !} L_{n}^{(-1 / 2)}(-1), r_{n}=\sqrt{\pi} 2^{2 n+2}(2 n+2) ! a_{n} a_{n+1}$ and note the simple appraisals: with $c=(4 \pi e)^{-1}$,

$$
\begin{aligned}
r_{n}^{-1} & =\frac{1}{4 c \pi \sqrt{n}} e^{-4 \sqrt{n}}\left(1+O\left(n^{-1 / 2}\right)\right), \\
a_{n} a_{n+m} 2^{2 n+2 m}(2 n) ! & =c \sqrt{\pi} n^{-m-1 / 2} e^{4 \sqrt{n}}\left(1+O\left(m n^{-1 / 2}\right)\right),
\end{aligned}
$$


where the latter will be used for $m$ nonnegative and moderate (compared with $n^{1 / 2}$ ). We will also make repeated use of the fact

$$
\sum_{k=1}^{n} n^{m-1 / 2} e^{4 \sqrt{n}}=\frac{1}{2} n^{m} e^{4 \sqrt{n}}\left(1+O\left(n^{-1 / 2}\right)\right)
$$

valid for any real $m$.

Continuing, we change the order of summation to write

$$
\hat{s}_{N, d}^{(1)}=\frac{4 \sqrt{2 \pi}}{\sqrt{N}} \sum_{m=0}^{N-2} \frac{\left(-2 t^{2} / N\right)^{m}}{(m !)^{2}} \sum_{n=\lceil m / 2\rceil}^{N / 2-1} r_{n}^{-1} \sum_{k=\lceil m / 2\rceil}^{n}(2 k)_{m} a_{k}^{2} 2^{2 k}(2 k) ! .
$$

Then, for fixed $m$,

$$
\sum_{n=\lceil m / 2\rceil}^{N / 2-1} r_{n}^{-1} \sum_{k=\lceil m / 2\rceil}^{n}(2 k)_{m} a_{k}^{2} 2^{2 k}(2 k) !=\frac{N^{m+1 / 2}}{4 \sqrt{2 \pi}(2 m+1)}(1+o(1)),
$$

by (4.11) and (4.12), and a dominated convergence argument yields

$$
\lim _{N \rightarrow \infty} \hat{s}_{N, d}^{(1)}=\sum_{m=0}^{\infty} \frac{\left(-t^{2} / 2\right)^{m}}{(m !)^{2}(2 m+1)}=\int_{0}^{1} J_{0}(\sqrt{2} t x) d x,
$$

for the diagonal contribution. Next, for the off-diagonal terms (second line of (4.10)), we again change the order of summation and have $\hat{s}_{N, o}^{(1)}$ equal to

$$
\frac{8 \sqrt{2 \pi}}{\sqrt{N}} \sum_{q=1}^{N / 2-1} \sum_{m=0}^{N-2 q-2} \frac{\left(-2 t^{2} / N\right)^{q+m}}{m !(2 q+m) !} \sum_{n=q+\lceil m / 2\rceil}^{N / 2-1} r_{n}^{-1} \sum_{k=\lceil m / 2\rceil}^{n-q}(2 k+2 q)_{2 q+m} a_{k} a_{k+q}(2 k) ! 2^{2 k+q} .
$$

With now $q$ and $m$ fixed,

$$
\begin{aligned}
& \sum_{n=q+\lceil m / 2\rceil}^{N / 2-1} r_{n}^{-1} \sum_{k=\lceil m / 2\rceil}^{n-q}(2 k+2 q)_{2 q+m} a_{k} a_{k+q}(2 k) ! 2^{2 k+q} \\
& =\frac{2^{q+m}}{4 \sqrt{\pi}} \sum_{n=1}^{N / 2} \frac{1}{\sqrt{n}} e^{-4 \sqrt{n}} \sum_{k=1}^{n} k^{q+m-1 / 2} e^{4 \sqrt{k}}(1+o(1))=\frac{N^{q+m+1 / 2}}{4 \sqrt{2 \pi}(2 q+2 m+1)}(1+o(1)),
\end{aligned}
$$

again by (4.11) and (4.12). Hence, for bounded $t$,

$$
\begin{aligned}
\hat{s}_{N, o}^{(1)} & =2 \sum_{q=1}^{N / 2-1} \sum_{m=0}^{N-2 q-2} \frac{\left(-t^{2} / 2\right)^{q+m}}{m !(2 q+m) !(2 q+2 m+1)}(1+o(1)) \\
& =2 \sum_{\ell=1}^{N} \frac{\left(-t^{2} / 2\right)^{\ell}}{(2 \ell+1)} \sum_{q=1}^{\ell} \frac{1}{(\ell-q) !(\ell+q) !}(1+o(1)) \\
& =\sum_{\ell=1}^{N} \frac{\left(-t^{2} / 2\right)^{\ell}}{(2 \ell+1)}\left(\frac{2^{2 \ell}}{(2 \ell) !}-\frac{1}{(\ell !)^{2}}\right)(1+o(1))
\end{aligned}
$$


after changing variables and the order of summation in line two. That is, $\hat{s}_{N, o}^{(1)}$ tends to $\frac{\sin \sqrt{2} t}{\sqrt{2} t}-\int_{0}^{1} J_{0}(\sqrt{2} t x) d x$, which, combined with (4.13), proves the first statement of the lemma.

Turning to (4.9), the preceding shows that, asymptotically, the $\left(x^{2}-\left(t_{N} / 2\right)^{2}\right)$ within the integrand may be replaced by $\frac{1}{4} H_{2}(x)=x^{2}-1 / 2$ for which there is the related evaluation: assuming $k \leq \ell$,

$$
\begin{aligned}
& \int_{-\infty}^{\infty} H_{2}(x) H_{2 k}(x+t / 2) H_{2 \ell}(x-t / 2) e^{-x^{2}} d x \\
& =4 \sqrt{\pi} \sum_{\substack{0 \leq n \leq 2 k \\
n-m=0, \pm 2}}\left(\begin{array}{c}
2 k \\
n
\end{array}\right)\left(\begin{array}{c}
2 \ell \\
m
\end{array}\right)(-1)^{m} \frac{n ! m ! 2^{\frac{n+m}{2}} t^{2 k+2 \ell-n-m}}{\left(\frac{n-m}{2}+1\right) !\left(\frac{m-n}{2}+1\right) !\left(\frac{n+m}{2}-1\right) !} .
\end{aligned}
$$

The resulting diagonal term (when $k=\ell$ in (4.9)) then reads

$$
\begin{aligned}
\hat{s}_{N, d}^{(2)}= & \frac{8 \sqrt{\pi}}{N} \sum_{n=1}^{N / 2-1} r_{n}^{-1} \sum_{k=1}^{n} a_{k}^{2} 2^{2 k}(2 k) ! \sum_{m=0}^{2 k} \frac{(2 k)_{m}(2 k-m)}{(m !)^{2}}\left(-\frac{t^{2}}{2 N}\right)^{m} \\
& -\frac{8 \sqrt{\pi}}{N} \sum_{n=1}^{N / 2-1} r_{n}^{-1} \sum_{k=1}^{n} a_{k}^{2} 2^{2 k}(2 k) ! \sum_{m=0}^{2 k-2} \frac{(2 k)_{m+2}}{m !(m+2) !}\left(-\frac{t^{2}}{2 N}\right)^{m+1} .
\end{aligned}
$$

This object does not converge on its own; cancellations from the off-diagonals are required.

With similar notation to the above we decompose $\hat{s}_{N, o}^{(2)}$ as in $\sum_{p \geq 1} \hat{s}_{N,(o,+p)}^{(2)}$ in which $\hat{s}_{N,(o,+p)}^{(2)}$ is arrived at by choosing $\ell=k+p$ in (4.9). Writing out the $p=1$ case in full we have that

$$
\begin{aligned}
\hat{s}_{N,(o,+1)}^{(2)}= & -\frac{16 \sqrt{\pi}}{N} \sum_{n=1}^{N / 2-1} r_{n}^{-1} \sum_{k=1}^{n-1} a_{k} a_{k+1} 2^{2 k}(2 k) ! \sum_{m=0}^{2 k} \frac{(2 k+2)_{m+2}}{(m !)^{2}}\left(-\frac{t^{2}}{2 N}\right)^{m} \\
& +\frac{32 \sqrt{\pi}}{N} \sum_{n=1}^{N / 2-1} r_{n}^{-1} \sum_{k=1}^{n-1} a_{k} a_{k+1} 2^{2 k}(2 k) ! \sum_{m=0}^{2 k-1} \frac{(2 k+2)_{m+2}(2 k-m)}{m !(m+2) !}\left(-\frac{t^{2}}{2 N}\right)^{m+1} \\
& -\frac{16 \sqrt{\pi}}{N} \sum_{n=1}^{N / 2-1} r_{n}^{-1} \sum_{k=1}^{n-1} a_{k} a_{k+1} 2^{2 k}(2 k) ! \sum_{m=0}^{2 k-2} \frac{(2 k+2)_{m+4}}{m !(m+4) !}\left(-\frac{t^{2}}{2 N}\right)^{m+2} .
\end{aligned}
$$

Consider now the first sum on the right of (4.15) for $k=n$ only:

$$
\begin{aligned}
& \frac{8 \sqrt{\pi}}{N} \sum_{n=1}^{N / 2-1} r_{n}^{-1} a_{n}^{2} 2^{2 n}(2 n) ! \sum_{m=0}^{2 n}(2 k)_{m}(2 n-m) \frac{\left(-t^{2} / 2 N\right)^{m}}{(m !)^{2}} \\
& =\frac{8 \sqrt{\pi}}{N} \sum_{m=0}^{N-2} \frac{\left(-t^{2} / 2 N\right)^{m}}{(m !)^{2}} \sum_{n=\lceil m / 2\rceil}^{N / 2-1} r_{n}^{-1} a_{n}^{2} 2^{2 n}(2 n) !(2 n)^{m+1}(1+o(1)) \\
& =2 \sum_{m=0}^{N} \frac{\left(-t^{2} / 2\right)^{m}}{(m !)^{2}(m+1)}(1+o(1)),
\end{aligned}
$$


by the same type of estimates used in the analysis of $\hat{s}_{N}^{(1)}$. Next, using the additional fact that $1-4 k a_{k+1} a_{k}^{-1}=-k^{-1 / 2}\left(1+O\left(k^{-1}\right)\right)$ the remainder (or $k \leq n-1$ part) of the first sum in (4.15) plus the first sum in (4.16) is asymptotic to

$$
\begin{gathered}
-\frac{8 \sqrt{\pi}}{N} \sum_{m=0}^{N-2} \frac{\left(-t^{2} / 2\right)^{m}}{(m !)^{2}} \sum_{n=\lceil m / 2\rceil}^{N / 2} r_{n}^{-1} \sum_{k=\lceil m / 2\rceil}^{n-1} a_{k}^{2} 2^{2 k}(2 k) ! \times \frac{1}{\sqrt{k}}(2 k)^{m+1} \\
=-\sum_{m=0}^{N} \frac{\left(-t^{2} / 2\right)^{m}}{(m !)^{2}(m+1)}(1+o(1)) .
\end{gathered}
$$

The last two displays combine to produce the advertised limit $\frac{\sqrt{2}}{t} J_{1}(\sqrt{2} t)$.

The above ideas propagate. In particular, the remaining terms of $\hat{s}_{N, d}^{(2)}+\hat{s}_{N,(o,+1)}^{(2)}$ balance to produce a $o(1)$ contribution, and this appraisal extends to the full sum over $p>1$ of $\hat{s}_{N,(o,+p)}^{(2)}$. We do not reproduce the details.

Revisiting second terms in (4.5) and (4.6) shows that the proof of Theorem 2.3 can be completed by the following (rough) overestimates.

Lemma 4.2. As $N \rightarrow \infty$,

$$
\sum_{n=0}^{N / 2-1} r_{n}^{-1} \int_{-\infty}^{\infty}\left|\widetilde{p}_{2 n}(x) \epsilon_{1} \widetilde{p}_{2 n}(x)\right| d x=O\left(N^{1 / 2}\right), \sum_{n=0}^{N / 2-1} r_{n}^{-1} \int_{-\infty}^{\infty}\left|\widetilde{p}_{2 n}(x) \widetilde{p}_{2 n+1}(x)\right| d x=O(N) .
$$

Proof. Along with the well known evaluation $\left\|\widetilde{H}_{k}\right\|_{L^{2}}=\pi^{1 / 4} 2^{n / 2} \sqrt{n !}$ used several time already, it holds that $\left\|\widetilde{H}_{k}\right\|_{L^{1}}=c 2^{n / 2} \sqrt{n !} n^{-1 / 4}\left(1+O\left(n^{-1}\right)\right.$ with a (known) numerical constant $c$. Next note that $\left\|f \epsilon_{1} g\right\|_{L^{1}} \leq\|f\|_{L^{1}}\|g\|_{L^{1}}$ and so

$$
\begin{aligned}
\left\|\widetilde{p}_{2 n} \epsilon_{1} \widetilde{p}_{2 n}\right\|_{L^{1}} \leq\left\|\widetilde{p}_{2 n}\right\|_{L^{1}}^{2} & =\left(\sum_{k=0}^{n} a_{k}\left\|\widetilde{H}_{2 k}\right\|_{L^{1}}\right)^{2} \\
& \leq C\left(\sum_{k=0}^{n} \frac{1}{\sqrt{k}} e^{2 \sqrt{k}}\right)^{2}=O\left(e^{4 \sqrt{n}}\right) .
\end{aligned}
$$

Here we have used, again, that $a_{k} 2^{k} \sqrt{2 k !} \sim k^{-1 / 4} e^{2 \sqrt{k}}$. Recalling that $r_{n}^{-1} \sim n^{-1 / 2} e^{4 \sqrt{n}}$ finishes the first part.

For the second estimate we can get by with an application of Schwarz's inequality. Simply compute

$$
\left\|\widetilde{p}_{2 n}\right\|_{L^{2}}^{2}=\sum_{k=0}^{n} a_{k}^{2}\left\|\widetilde{H}_{2 k}\right\|_{L^{2}}^{2} \leq C \sum_{k=0}^{n} \frac{1}{\sqrt{k}} e^{4 \sqrt{k}}=O\left(e^{4 \sqrt{n}}\right),
$$

and

$$
\begin{aligned}
\left\|\widetilde{p}_{2 n+1}\right\|_{L^{2}}^{2} & =\sum_{k=0}^{n} a_{k}^{2}\left(\left\|\widetilde{H}_{2 k+1}\right\|_{L^{2}}^{2}+16 k^{2}\left\|\widetilde{H}_{2 k-1}\right\|_{L^{2}}^{2}\right)+2 \sum_{k=0}^{n-1} a_{k} a_{k-1}\left\|\widetilde{H}_{2 k+1}\right\|_{L^{2}}^{2} \\
& \leq C \sum_{k=0}^{n} \sqrt{k} e^{4 \sqrt{k}}=O\left(n e^{4 \sqrt{n}}\right),
\end{aligned}
$$

to find that $r_{n}^{-1}|| \widetilde{p}_{2 n}\left\|_{L^{2}}\right\| \widetilde{p}_{2 n+1} \|_{L^{2}}=O(1)$, which suffices. 


\subsection{Proof of Theorem 3.1}

We will prove something slightly more general which will be useful in the sequel.

Given measures $\nu_{1}$ and $\nu_{2}$ on $\mathbb{R}$, define

$Z_{L, M}^{\nu_{1}, \nu_{2}}=\frac{1}{L ! M !} \int_{\mathbb{R}^{L}} \int_{\mathbb{R}^{M}}\left\{\prod_{j<k}\left|\alpha_{j}-\alpha_{k}\right| \prod_{m<n}\left|\beta_{m}-\beta_{n}\right|^{4} \prod_{\ell=1}^{L} \prod_{m=1}^{M}\left|\alpha_{\ell}-\beta_{m}\right|^{2}\right\} d \nu_{1}^{L}(\alpha) d \nu_{2}^{M}(\beta)$,

and

$$
Z^{\nu_{1}, \nu_{2}}(X)=\sum_{(L, M)} X^{L} Z_{L, M}^{\nu_{1}, \nu_{2}}
$$

Theorem 4.3. Suppose $N$ is even and $\mathbf{p}$ is any complete family of monic polynomials.

$$
Z^{\nu_{1}, \nu_{2}}(X)=\operatorname{Pf}\left(X^{2} \mathbf{A}^{\mathbf{p}}+\mathbf{B}^{\mathbf{p}}\right)
$$

where

$$
\mathbf{A}^{\mathbf{p}}=\left[\left\langle p_{m} \mid p_{n}\right\rangle_{1}^{\nu_{1}}\right]_{m, n=0}^{N-1} \quad \text { and } \quad \mathbf{B}^{\mathbf{p}}=\left[\left\langle p_{m} \mid p_{n}\right\rangle_{4}^{\nu_{2}}\right]_{m, n=0}^{N-1}
$$

\subsubsection{The Confluent Vandermonde Determinant}

A special case of the confluent Vandermonde determinant identity has that

$$
\begin{gathered}
\operatorname{det}\left[\begin{array}{cccccccc}
1 & & 1 & 1 & 0 & & 1 & 0 \\
\alpha_{1} & & \alpha_{L} & \beta_{1} & 1 & & \beta_{M} & 1 \\
\alpha_{1}^{2} & \cdots & \alpha_{L}^{2} & \beta_{1}^{2} & 2 \beta_{1} & \cdots & \beta_{M}^{2} & 2 \beta_{M} \\
\vdots & \ddots & \vdots & & \vdots & \ddots & \vdots & \\
\alpha_{1}^{N-1} & \cdots & \alpha_{L}^{N-1} & \beta_{1}^{N-1} & (N-1) \beta_{1}^{N-2} & \cdots & \beta_{M}^{N-1} & (N-1) \beta_{M}^{N-2}
\end{array}\right] \\
\\
\quad=\prod_{j<k}\left(\alpha_{k}-\alpha_{j}\right) \prod_{m<n}\left(\beta_{m}-\beta_{n}\right)^{4} \prod_{\ell=1}^{L} \prod_{m=1}^{M}\left(\alpha_{\ell}-\beta_{m}\right)^{2} .
\end{gathered}
$$

We will denote the matrix on the left hand side of (4.20) by $\mathbf{V}(\boldsymbol{\alpha}, \boldsymbol{\beta})$ and its determinant by $\Delta(\boldsymbol{\alpha}, \boldsymbol{\beta})$. We will later use the fact that the monomials which appear in the definition of $\mathbf{V}(\boldsymbol{\alpha}, \boldsymbol{\beta})$ can be replaced by any family of monic polynomials $\mathbf{p}=\left(p_{0}, p_{2}, \ldots, p_{N-1}\right)$ with $\operatorname{deg} p_{n}=n$ without changing the determinant. We will write the resulting matrix $\mathbf{V}^{\mathbf{p}}(\boldsymbol{\alpha}, \boldsymbol{\beta})$, and we note that $\Delta(\boldsymbol{\alpha}, \boldsymbol{\beta})=\operatorname{det} \mathbf{V}^{\mathbf{p}}(\boldsymbol{\alpha}, \boldsymbol{\beta})$.

It follows from (1.2) and (1.3) that

$$
Z_{L, M}^{\nu_{1}, \nu_{2}}=\frac{1}{L ! M !} \int_{\mathbb{R}^{L}} \int_{\mathbb{R}^{M}}\left|\operatorname{det} \mathbf{V}^{\mathbf{p}}(\boldsymbol{\alpha}, \boldsymbol{\beta})\right| d \nu_{1}^{L}(\alpha) d \nu_{2}^{M}(\beta) .
$$

\subsubsection{Notation for minors}

Given a non-negative integer $L$, we denote the set $\{1,2, \ldots, L\}$ by $\underline{L}$. By convention, if $L=0$, then $\underline{L}$ is the empty set. Given a function $\mathfrak{t}: \underline{L} \nearrow \underline{N}$ we denote by $\mathfrak{t}^{\prime}$ the unique function $\underline{N-L} \nearrow \underline{N}$ whose range is disjoint from $\mathfrak{t}$. We denote by $\mathfrak{i}$ the function $\underline{L} \nearrow \underline{N}$ which is the identity on $\underline{L}$. 
We define sgn $\mathfrak{t}$ as follows: Let $\mathbf{e}_{1}, \mathbf{e}_{2}, \ldots, \mathbf{e}_{N}$ be any particular basis for $\mathbb{R}^{N}$. We then specify that

$$
\mathbf{e}_{\mathfrak{t}(1)} \wedge \cdots \wedge \mathbf{e}_{\mathfrak{t}(L)} \wedge \mathbf{e}_{\mathfrak{t}^{\prime}(1)} \wedge \cdots \wedge \mathbf{e}_{\mathfrak{t}^{\prime}(N-L)}=\operatorname{sgn} \mathfrak{t} \cdot \mathbf{e}_{1} \wedge \mathbf{e}_{2} \wedge \cdots \wedge \mathbf{e}_{N} .
$$

That is $\operatorname{sgn} \mathfrak{t}=(-1)^{k}$ where $k$ is the number of transpositions necessary to put the set

$$
\mathfrak{t}(1), \cdots \mathfrak{t}(L), \mathfrak{t}^{\prime}(1), \cdots, \mathfrak{t}^{\prime}(N-L)
$$

into order. Clearly, $\operatorname{sgn} \mathfrak{i}=1$.

Given an increasing function $\mathfrak{t}: \underline{L} \nearrow \underline{N}$ and a vector $\alpha \in \mathbb{R}^{N}$, we define the vector $\boldsymbol{\alpha}_{\mathfrak{t}} \in \mathbb{R}^{L}$ by

$$
\boldsymbol{\alpha}_{\mathfrak{t}}=\left(\alpha_{\mathfrak{t}(1)}, \alpha_{\mathfrak{t}(2)}, \ldots, \alpha_{\mathfrak{t}(L)}\right)
$$

If $\mathfrak{u}: \underline{L} \nearrow \underline{N}$ is another increasing function, and $\mathbf{A}=\left[a_{m, n}\right]$ an $N \times N$ matrix , we define $\mathbf{A}_{\mathfrak{t}, \mathfrak{u}}$ to be the $L \times L$ minor of $\mathbf{A}$ given by

$$
\mathbf{A}_{\mathfrak{t}, \mathfrak{u}}=\left[a_{\mathfrak{t}(j), \mathfrak{u}(k)}\right]_{j, k=1}^{L} .
$$

\subsubsection{The Laplace expansion of the determinant}

Using this notation, the Laplace expansion of the determinant is given by

$$
\operatorname{det} \mathbf{A}=\sum_{\mathfrak{t}: \underline{L} \nearrow \underline{N}} \operatorname{sgn} \mathfrak{t} \cdot \operatorname{det} \mathbf{A}_{\mathfrak{t}, \mathfrak{i}} \cdot \operatorname{det} \mathbf{A}_{\mathfrak{t}^{\prime}, \mathfrak{i}^{\prime}} \cdot
$$

In particular, the Laplace expansion of the determinant of $\mathbf{V}^{\mathbf{p}}(\boldsymbol{\alpha}, \boldsymbol{\beta})$ is given by

$$
\mathbf{V}^{\mathbf{p}}(\boldsymbol{\alpha}, \boldsymbol{\beta})=\sum_{\mathfrak{t}: \underline{L} \nearrow \underline{N}} \operatorname{sgn} \mathfrak{t} \cdot \operatorname{det} \mathbf{V}_{\mathfrak{t}, \mathfrak{i}}^{\mathbf{p}}(\boldsymbol{\alpha}) \cdot \operatorname{det} \mathbf{V}_{\mathfrak{t}^{\prime}, \mathfrak{i}^{\prime}}(\boldsymbol{\beta}),
$$

where the notation reflects the fact that minors of the form $\mathbf{V}_{\mathfrak{t}, \mathbf{i}}$ depend only on $\boldsymbol{\alpha}$ and minors of the form $\mathbf{V}_{\mathfrak{t}^{\prime}, i^{\prime}}$ only depend on $\boldsymbol{\beta}$.

\subsubsection{The Total Partition Function}

Using the previous definitions, we may write

$$
\left|\operatorname{det} \mathbf{V}^{\mathbf{p}}(\boldsymbol{\alpha}, \boldsymbol{\beta})\right|=\sum_{\mathfrak{t}: \underline{\underline{y}} \underline{\underline{N}}} \operatorname{sgn} \mathfrak{t}\left\{\prod_{j<k} \operatorname{sgn}\left(\alpha_{k}-\alpha_{j}\right)\right\} \operatorname{det} \mathbf{V}_{\mathbf{t}, \mathfrak{i}}^{\mathbf{p}}(\boldsymbol{\alpha}) \operatorname{det} \mathbf{V}_{\mathfrak{t}^{\prime}, \mathfrak{i}^{\prime}}^{\mathbf{p}}(\boldsymbol{\beta}),
$$

and

$$
\begin{gathered}
Z_{L, M}^{\nu_{1}, \nu_{2}}=\sum_{\mathfrak{t}: \underline{\underline{L} \nearrow \underline{N}}} \operatorname{sgn} \mathfrak{t} \frac{1}{L !} \int_{\mathbb{R}^{L}}\left\{\prod_{j<k} \operatorname{sgn}\left(\alpha_{k}-\alpha_{j}\right)\right\} \operatorname{det} \mathbf{V}_{\mathfrak{t}, \mathfrak{i}}^{\mathbf{p}}(\boldsymbol{\alpha}) d \nu_{1}^{L}(\boldsymbol{\alpha}) \\
\times \frac{1}{M !} \int_{\mathbb{R}^{M}} \operatorname{det} \mathbf{V}_{\mathfrak{t}^{\prime}, \mathfrak{i}^{\prime}}^{\mathbf{p}}(\boldsymbol{\beta}) d \nu_{2}^{M}(\boldsymbol{\beta}) .
\end{gathered}
$$

We define

$$
A_{\mathfrak{t}}=\frac{1}{L !} \int_{\mathbb{R}^{L}}\left\{\prod_{j<k} \operatorname{sgn}\left(\alpha_{k}-\alpha_{j}\right)\right\} \operatorname{det} \mathbf{V}_{\mathfrak{t}, \mathrm{i}}^{\mathbf{p}}(\boldsymbol{\alpha}) d \nu_{1}^{L}(\boldsymbol{\alpha})
$$


and

$$
B_{\mathfrak{t}^{\prime}}=\frac{1}{M !} \int_{\mathbb{R}^{M}} \operatorname{det} \mathbf{V}_{\mathfrak{t}^{\prime}, \mathfrak{i}^{\prime}}^{\mathbf{p}}(\boldsymbol{\beta}) d \nu_{2}^{M}(\boldsymbol{\beta})
$$

so that

$$
Z_{L, M}^{\nu_{1}, \nu_{2}}=\sum_{\mathfrak{t}: \underline{L} \nearrow \underline{N}} \operatorname{sgn} \mathfrak{t} \cdot A_{\mathfrak{t}} B_{\mathfrak{t}^{\prime}}
$$

\subsubsection{Simplifying $A_{\mathfrak{t}}$}

If $L$ is even,

$$
\prod_{j<k} \operatorname{sgn}\left(\alpha_{k}-\alpha_{j}\right)=\operatorname{Pf} \mathbf{T}(\boldsymbol{\alpha})
$$

where $\mathbf{T}(\boldsymbol{\alpha})$ is the $L \times L$ antisymmetric matrix given by

$$
\mathbf{T}(\boldsymbol{\alpha})=\left[\operatorname{sgn}\left(\alpha_{k}-\alpha_{j}\right)\right]_{j, k=1}^{L} .
$$

A similar formula is available when $L$ is odd, but since $N$ and therefore $L$ is even, we will not need that here.

It follows that

$$
A_{\mathfrak{t}}=\frac{1}{L !} \int_{\mathbb{R}^{L}} \operatorname{Pf} \mathbf{T}(\boldsymbol{\alpha}) \cdot \operatorname{det} \mathbf{V}_{\mathfrak{t}, \mathfrak{i}}^{\mathbf{p}}(\boldsymbol{\alpha}) d \nu_{1}^{L}(\boldsymbol{\alpha})
$$

and

$$
\operatorname{det} \mathbf{V}_{\mathfrak{t}, \mathfrak{i}}^{\mathbf{p}}(\boldsymbol{\alpha})=\sum_{\sigma \in S_{L}} \operatorname{sgn} \sigma \prod_{\ell=1}^{L} p_{\mathfrak{t}(\ell)-1}\left(\alpha_{\sigma(\ell)}\right)
$$

where $S_{L}$ is the symmetric group of $L$ elements, so that,

$$
A_{\mathfrak{t}}=\frac{1}{L !} \sum_{\sigma \in S_{L}} \int_{\mathbb{R}^{L}}\left\{p_{\mathfrak{t}(\ell)-1}\left(\alpha_{\sigma(\ell)}\right)\right\} \operatorname{sgn} \sigma \cdot \operatorname{Pf} \mathbf{T}(\boldsymbol{\alpha}) d \nu_{1}^{L}(\boldsymbol{\alpha}) .
$$

Now, $S_{L}$ acts on $\mathbb{R}^{L}$ by permuting coordinates - denote the action of $\sigma$ by $\boldsymbol{\alpha} \mapsto \sigma \cdot \boldsymbol{\alpha}$. It is easy to verify that

$$
\operatorname{Pf} \mathbf{T}(\sigma \cdot \boldsymbol{\alpha})=\operatorname{sgn} \sigma \cdot \operatorname{Pf} \mathbf{T}(\boldsymbol{\alpha})
$$

Consequently,

$$
A_{\mathfrak{t}}=\frac{1}{L !} \sum_{\sigma \in S_{L}} \int_{\mathbb{R}^{L}}\left\{\prod_{\ell=1}^{L} p_{\mathfrak{t}(\ell)-1}\left(\alpha_{\sigma(\ell)}\right)\right\} \operatorname{Pf} \mathbf{T}(\sigma \cdot \boldsymbol{\alpha}) d \nu_{1}^{L}(\boldsymbol{\alpha})
$$

and by reindexing the integral by $\boldsymbol{\alpha} \mapsto \sigma^{-1} \cdot \boldsymbol{\alpha}$, we find that

$$
A_{\mathfrak{t}}=\frac{1}{L !} \sum_{\sigma \in S_{L}} \int_{\mathbb{R}^{L}}\left\{\prod_{\ell=1}^{L} p_{\mathfrak{t}(\ell)-1}\left(\alpha_{\ell}\right)\right\} \operatorname{Pf} \mathbf{T}(\boldsymbol{\alpha}) d \nu_{1}^{L}(\boldsymbol{\alpha})=\int_{\mathbb{R}^{L}}\left\{\prod_{\ell=1}^{L} p_{\mathfrak{t}(\ell)-1}\left(\alpha_{\ell}\right)\right\} \operatorname{Pf} \mathbf{T}(\boldsymbol{\alpha}) d \nu_{1}^{L}(\boldsymbol{\alpha})
$$

Next, we write $L=2 K$ and expand $\operatorname{Pf} \mathbf{T}(\boldsymbol{\alpha})$ as a sum over the symmetric group:

$$
\operatorname{Pf} \mathbf{T}(\boldsymbol{\alpha})=\frac{1}{2^{K} K !} \sum_{\tau \in S_{L}} \operatorname{sgn} \tau \prod_{k=1}^{K} \operatorname{sgn}\left(\alpha_{\tau(2 k)}-\alpha_{\tau(2 k-1)}\right),
$$


so that

$$
\begin{aligned}
A_{\mathfrak{t}}= & \frac{1}{2^{K} K !} \sum_{\tau \in S_{L}} \operatorname{sgn} \tau \int_{\mathbb{R}^{L}}\left\{\prod_{k=1}^{K} p_{\mathfrak{t}(2 k-1)-1}\left(\alpha_{2 k-1}\right) p_{\mathfrak{t}(2 k)-1}\left(\alpha_{2 k}\right) \operatorname{sgn}\left(\alpha_{\tau(2 k)}-\alpha_{\tau(2 k-1)}\right)\right\} d \nu_{1}^{L}(\boldsymbol{\alpha}) \\
= & \frac{1}{2^{K} K !} \sum_{\tau \in S_{L}} \operatorname{sgn} \tau \\
& \quad \times \int_{\mathbb{R}^{L}}\left\{\prod_{k=1}^{K} p_{\mathfrak{t o} \tau(2 k-1)-1}\left(\alpha_{\tau(2 k-1)}\right) p_{\mathrm{to} \tau(2 k)-1}\left(\alpha_{\tau(2 k)}\right) \operatorname{sgn}\left(\alpha_{\tau(2 k)}-\alpha_{\tau(2 k-1)}\right)\right\} d \nu_{1}^{L}(\boldsymbol{\alpha}) \\
= & \frac{1}{2^{K} K !} \sum_{\tau \in S_{L}} \operatorname{sgn} \tau \prod_{k=1}^{K} \int_{\mathbb{R}^{2}} p_{\mathfrak{t o} \tau(2 k-1)-1}(x) p_{\mathfrak{t o} \tau(2 k)-1}(y) \operatorname{sgn}(y-x) d \nu_{1}(x) d \nu_{1}(y)=\operatorname{Pf} \mathbf{A}_{\mathfrak{t}}^{\mathbf{p}} .
\end{aligned}
$$

\subsubsection{Simplifying $B_{\mathfrak{t}^{\prime}}$}

Turning to $B_{\mathfrak{t}^{\prime}}$, we first write

$$
\operatorname{det} \mathbf{V}_{\mathfrak{t}^{\prime}, \mathfrak{i}^{\prime}}^{\mathbf{p}}(\boldsymbol{\beta})=\sum_{\sigma \in S_{2 M}} \operatorname{sgn} \sigma \prod_{m=1}^{M} p_{\mathfrak{t}^{\prime} \circ \sigma(2 m-1)-1}\left(\beta_{m}\right) p_{\mathbf{t}^{\prime} \circ \sigma(2 m)-1}^{\prime}\left(\beta_{m}\right),
$$

so that

$$
\begin{aligned}
B_{\mathfrak{t}^{\prime}} & =\frac{1}{M !} \sum_{\sigma \in S_{2 M}} \operatorname{sgn} \sigma \int_{\mathbb{R}^{M}}\left\{\prod_{m=1}^{M} p_{\mathfrak{t}^{\prime} \circ \sigma(2 m-1)-1}\left(\beta_{m}\right) p_{\mathfrak{t}^{\prime} \circ \sigma(2 m)-1}^{\prime}\left(\beta_{m}\right)\right\} d \nu_{2}^{M}(\boldsymbol{\beta}) \\
& =\frac{1}{M !} \sum_{\sigma \in S_{2 M}} \operatorname{sgn} \sigma \prod_{m=1}^{M} \int_{\mathbb{R}} p_{\mathfrak{t}^{\prime} \circ \sigma(2 m-1)-1}(\beta) p_{\mathfrak{t}^{\prime} \circ \sigma(2 m)-1}^{\prime}(\beta) d \nu_{2}(\beta) .
\end{aligned}
$$

Next we set

$$
\Pi_{2 M}=\left\{\sigma \in S_{2 M}: \sigma(2 m-1)<\sigma(2 m) \text { for } m=1,2, \ldots, M\right\},
$$

then

$$
\begin{aligned}
B_{\mathfrak{t}^{\prime}} & =\frac{1}{M !} \sum_{\sigma \in \Pi_{2 M}} \operatorname{sgn} \sigma \prod_{m=1}^{M} \int_{\mathbb{R}}\left[p_{\mathfrak{t}^{\prime} \circ \sigma(2 m-1)-1}(\beta) p_{\mathbf{t}^{\prime} \circ \sigma(2 m)}^{\prime}(\beta)-p_{\mathbf{t}^{\prime} \circ \sigma(2 m)}(\beta) p_{\mathfrak{t}^{\prime} \circ \sigma(2 m-1)-1}^{\prime}(\beta)\right] d \nu_{2}(\beta) \\
& =\operatorname{Pf} \mathbf{B}_{\mathbf{t}^{\prime}}^{\mathbf{P}} .
\end{aligned}
$$

\subsubsection{The Pfaffian formulation for the total partition function}

It follows from (4.21) that

$$
Z_{L, M}^{\nu_{1}, \nu_{2}}=\sum_{\mathfrak{t}: \underline{\underline{y}} \nearrow \underline{N}} \operatorname{sgn} \mathfrak{t} \cdot \operatorname{Pf} \mathbf{A}_{\mathfrak{t}}^{\mathbf{p}} \cdot \operatorname{Pf} \mathbf{B}_{\mathfrak{t}^{\prime}}^{\mathbf{p}}
$$

and therefore that,

$$
\begin{aligned}
Z^{\nu_{1}, \nu_{2}}(X) & =\sum_{(L, M)} X^{L} \sum_{\mathfrak{t}: \underline{L} \nearrow \underline{N}} \operatorname{sgn} \mathfrak{t} \cdot \operatorname{Pf} \mathbf{A}_{\mathfrak{t}}^{\mathbf{p}} \cdot \operatorname{Pf} \mathbf{B}_{\mathfrak{t}^{\prime}}^{\mathbf{p}} \\
& =\sum_{(L, M)} \sum_{\mathfrak{t}: \underline{L} \nearrow_{\underline{N}}} \operatorname{sgn} \mathfrak{t} \cdot X^{L} \operatorname{Pf} \mathbf{A}_{\mathfrak{t}}^{\mathbf{p}} \cdot \operatorname{Pf} \mathbf{B}_{\mathfrak{t}^{\prime}}^{\mathbf{p}} .
\end{aligned}
$$


Next, we set $\mathbf{X}=X \mathbf{I}$ where $\mathbf{I}$ is the $N \times N$ identity matrix. It is clear that, for each $\mathfrak{t}: \underline{L} \nearrow \underline{N}$,

$$
\left(\mathbf{X A}^{\mathrm{p}} \mathbf{X}^{\top}\right)_{\mathbf{t}}=\mathbf{X}_{\mathbf{t}} \mathbf{A}_{\mathfrak{t}}^{\mathrm{p}} \mathbf{X}_{\mathbf{t}}^{\top}
$$

and

$$
\operatorname{Pf}\left(\mathbf{X}_{\mathbf{t}} \mathbf{A}_{\mathfrak{t}}^{\mathbf{p}} \mathbf{X}_{\mathbf{t}}^{\boldsymbol{\top}}\right)=\operatorname{det} \mathbf{X}_{\mathfrak{t}} \cdot \operatorname{Pf} \mathbf{A}_{\mathfrak{t}}^{\mathbf{p}}=X^{L} \operatorname{Pf} \mathbf{A}_{\mathfrak{t}}^{\mathbf{p}}
$$

It follows that

$$
Z^{\nu_{1}, \nu_{2}}(X)=\sum_{(L, M)} \sum_{\mathfrak{t}: \underline{L} \nearrow \underline{N}} \operatorname{sgn} \mathfrak{t} \cdot \operatorname{Pf}\left(\mathbf{X A}^{\mathbf{p}} \mathbf{X}^{\mathbf{\top}}\right)_{\mathbf{t}} \cdot \operatorname{Pf} \mathbf{B}_{\mathfrak{t}^{\prime}}^{\mathbf{p}}=\operatorname{Pf}\left(\mathbf{X} \mathbf{A}^{\mathbf{p}} \mathbf{X}^{\boldsymbol{\top}}+\mathbf{B}^{\mathbf{p}}\right)
$$

where the last equation comes from the formula for the Pfaffian of a sum of antisymmetric matrices [10]. Finally, since $\mathbf{X} \mathbf{A}^{\mathbf{p}} \mathbf{X}^{\top}=X^{2} \mathbf{A}^{\mathbf{p}}$, we arrive at Theorem 4.3

\subsection{Proof of Theorem 3.3}

Given $x_{1}, \ldots, x_{N}, y_{1}, \ldots, y_{N} \in \mathbb{R}$ and indeterminants $a_{1}, \ldots, a_{N}, b_{1}, \ldots, b_{N}$ we define the measures $\eta_{1}$ and $\eta_{2}$ on $\mathbb{R}$ given by

$$
d \eta_{1}(\alpha)=\sum_{n=1}^{N} a_{n} d \delta\left(\alpha-x_{n}\right) \quad \text { and } \quad d \eta_{2}(\beta)=\sum_{n=1}^{N} b_{n} d \delta\left(\beta-y_{n}\right),
$$

where $\delta$ is the probability measure with unit point mass at 0 . Using these measures, we will specialize the situation in Section 4.4 to the measures $\nu_{1}=w\left(\mu+\eta_{1}\right)$ and $\nu_{2}=w^{2}\left(\mu+\eta_{2}\right)$. We will derive a Pfaffian form for the correlation functions of the microcanonical ensemble with weight $w$ by expanding both the integral and Pfaffian sides of (4.19) for this choice of $\nu_{1}$ and $\nu_{2}$ and equating coefficients of the various products of the indeterminants.

\subsubsection{Expanding the Integral Definition of $Z^{\nu_{1}, \nu_{2}}$}

Starting with (4.17), and setting $Z^{\nu_{1}, \nu_{2}}=Z^{\nu_{1}, \nu_{2}}(1)$, we have

$$
\frac{Z^{\nu_{1}, \nu_{2}}}{Z}=\sum_{(L, M)} \frac{1}{L ! M !} \int_{\mathbb{R}^{L}} \int_{\mathbb{R}^{M}} \Omega_{L, M}(\boldsymbol{\alpha}, \boldsymbol{\beta}) d\left(\mu+\eta_{1}\right)^{L}(\boldsymbol{\alpha}) d\left(\mu+\eta_{2}\right)^{M}(\boldsymbol{\beta}) .
$$

It is easily verified that

$$
d\left(\mu+\eta_{1}\right)^{L}(\boldsymbol{\alpha})=\sum_{\ell=0}^{L} \sum_{\mathfrak{u}: \underline{\ell} \underline{L}} d \eta_{1}^{\ell}\left(\boldsymbol{\alpha}_{\mathfrak{u}}\right) d \mu^{L-\ell}\left(\boldsymbol{\alpha}_{\mathfrak{u}^{\prime}}\right)
$$

and

$$
d\left(\mu+\eta_{2}\right)^{M}(\boldsymbol{\beta})=\sum_{m=0}^{M} \sum_{\mathfrak{v}: \underline{m} \nearrow \underline{M}} d \eta_{2}^{m}\left(\boldsymbol{\beta}_{\mathfrak{v}}\right) d \mu^{M-m}\left(\boldsymbol{\beta}_{\mathfrak{v}^{\prime}}\right)
$$

so that,

$$
\begin{aligned}
\frac{Z^{\nu_{1}, \nu_{2}}}{Z}=\sum_{(L, M)} \sum_{\ell=0}^{L} \sum_{m=0}^{M} & \sum_{\mathfrak{u}: \underline{\ell} \nearrow \underline{\underline{L}}} \sum_{\mathfrak{v}: \underline{m} \nearrow \underline{M}} \frac{1}{L ! M !} \\
& \times \int_{\mathbb{R}^{L}} \int_{\mathbb{R}^{M}} \Omega_{L, M}(\boldsymbol{\alpha}, \boldsymbol{\beta}) d \eta_{1}^{\ell}\left(\boldsymbol{\alpha}_{\mathfrak{u}}\right) d \mu^{L-\ell}\left(\boldsymbol{\alpha}_{\mathfrak{u}^{\prime}}\right) d \eta_{2}^{m}\left(\boldsymbol{\beta}_{\mathfrak{v}}\right) d \mu^{M-m}\left(\boldsymbol{\beta}_{\mathfrak{v}^{\prime}}\right) .
\end{aligned}
$$


We may relabel the $\alpha$ and the $\beta$ in any manner we see fit. In particular, we may replace each $\mathfrak{u}: \underline{\ell} \nearrow \underline{L}$ and $\mathfrak{v}: \underline{m} \nearrow \underline{M}$ by $\mathfrak{i}: \underline{\ell} \nearrow \underline{L}$ and $\mathfrak{i}: \underline{m} \nearrow \underline{M}$ respectively (the redundancy in the notation should cause no confusion). We compensate by a factor of $\left(\begin{array}{l}L \\ \ell\end{array}\right)\left(\begin{array}{l}M \\ m\end{array}\right)$ which counts the number of pairs $(\mathfrak{u}, \mathfrak{v})$ we are summing over. That is,

$$
\begin{aligned}
\frac{Z^{\nu_{1}, \nu_{2}}}{Z}=\sum_{(L, M)} \sum_{\ell=0}^{L} \sum_{m=0}^{M} \frac{1}{\ell !(L-\ell) ! m !(M-m) !} \\
\quad \times \int_{\mathbb{R}^{L}} \int_{\mathbb{R}^{M}} \Omega_{L, M}(\boldsymbol{\alpha}, \boldsymbol{\beta}) d \eta_{1}^{\ell}\left(\boldsymbol{\alpha}_{\mathfrak{i}}\right) d \mu^{L-\ell}\left(\boldsymbol{\alpha}_{\mathfrak{i}^{\prime}}\right) d \eta_{2}^{m}\left(\boldsymbol{\beta}_{\mathfrak{i}}\right) d \mu^{M-m}\left(\boldsymbol{\beta}_{\mathfrak{i}^{\prime}}\right) .
\end{aligned}
$$

Now,

$$
d \eta_{1}^{\ell}\left(\boldsymbol{\alpha}_{\mathfrak{i}}\right)=\prod_{j=1}^{\ell} \sum_{\mathfrak{u}=1}^{N} a_{u} d \delta\left(\alpha_{j}-x_{u}\right) \quad \text { and } \quad d \eta_{2}^{\ell}\left(\boldsymbol{\beta}_{\mathfrak{i}}\right)=\prod_{k=1}^{m} \sum_{v=1}^{N} b_{v} d \delta\left(\beta_{k}-y_{v}\right),
$$

and exchanging the sum and product in each of these expressions,

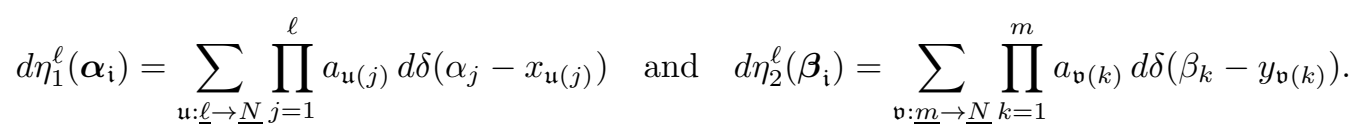

Notice that the sums in the latter two expressions are over all (not only increasing) functions from $\underline{\ell}$ and $\underline{m}$ into $\underline{N}$.

So far, we have that

$$
\begin{aligned}
& \frac{Z^{\nu_{1}, \nu_{2}}}{Z}=\sum_{(L, M)} \sum_{\ell=0}^{L} \sum_{m=0}^{M} \sum_{\mathfrak{u}: \underline{\underline{\ell} \rightarrow \underline{N}}} \sum_{\mathfrak{v}: \underline{m} \rightarrow \underline{N}} \frac{1}{\ell !(L-\ell) ! m !(M-m) !} \int_{\mathbb{R}^{L}} \int_{\mathbb{R}^{M}} \Omega_{L, M}(\boldsymbol{\alpha}, \boldsymbol{\beta}) \\
& \times\left\{\prod_{j=1}^{\ell} a_{\mathfrak{u}(j)} d \delta\left(\alpha_{j}-x_{\mathfrak{u}(j)}\right)\right\} d \mu^{L-\ell}\left(\boldsymbol{\alpha}_{\mathfrak{i}^{\prime}}\right)\left\{\prod_{k=1}^{m} b_{\mathfrak{v}(k)} d \delta\left(\beta_{k}-y_{\mathfrak{v}(k)}\right)\right\} d \mu^{M-m}\left(\boldsymbol{\beta}_{\mathfrak{i}^{\prime}}\right) \\
& =\sum_{(L, M)} \sum_{\ell=0}^{L} \sum_{m=0}^{M} \sum_{\mathfrak{u}: \underline{\ell \rightarrow \underline{N}}} \sum_{\mathfrak{v}: \underline{m} \rightarrow \underline{N}} \frac{1}{\ell !(L-\ell) ! m !(M-m) !}\left\{\prod_{j=1}^{\ell} a_{\mathfrak{u}(j)} \prod_{k=1}^{m} b_{\mathfrak{v}(k)}\right\} \\
& \times \int_{\mathbb{R}^{L-\ell}} \int_{\mathbb{R}^{M-m}} \Omega_{L, M}\left(\mathbf{x}_{\mathfrak{u}} \vee \boldsymbol{\alpha}, \mathbf{y}_{\mathfrak{v}} \vee \boldsymbol{\beta}\right) d \mu^{L-\ell}\left(\boldsymbol{\alpha}_{\mathfrak{i}^{\prime}}\right) d \mu^{M-m}\left(\boldsymbol{\beta}_{\mathfrak{i}^{\prime}}\right) .
\end{aligned}
$$

Next we notice that, if $\mathfrak{u}$ or $\mathfrak{v}$ are not injective,

$$
\Omega_{L, M}\left(\mathbf{x}_{\mathfrak{u}} \vee \boldsymbol{\alpha}, \mathbf{y}_{\mathfrak{v}} \vee \boldsymbol{\beta}\right)=0,
$$

and we can therefore replace the sums over $\underline{\ell} \rightarrow \underline{N}$ and $\underline{m} \rightarrow \underline{N}$ with sums over $\underline{\ell} \hookrightarrow \underline{N}$ and $\underline{m} \hookrightarrow \underline{N}$. In fact, since $\Omega_{L, M}\left(\mathbf{x}_{\mathfrak{u}} \vee \boldsymbol{\alpha}, \mathbf{y}_{\mathfrak{v}} \vee \boldsymbol{\beta}\right)$ is symmetric in the coordinates of $\mathbf{x}_{\mathfrak{u}}$ and $\mathbf{y}_{\mathfrak{v}}$, we may replace these sums with sums over $\underline{\ell} \nearrow \underline{N}$ and $\underline{m} \nearrow \underline{N}$ so long as we compensate by factors of $\ell$ ! and $m$ !. Putting these observations with the definition of $R_{\ell, m}$, we arrive at the fact that

$$
\frac{Z^{\nu_{1}, \nu_{2}}}{Z}=\sum_{(L, M)} \sum_{\ell=0}^{L} \sum_{m=0}^{M} \sum_{\mathfrak{u}: \underline{\ell} \nearrow \underline{N} \underline{\mathfrak{v}: \underline{\underline{m}} \nearrow \underline{N}}}\left\{\prod_{j=1}^{\ell} a_{\mathfrak{u}(j)} \prod_{k=1}^{m} b_{\mathfrak{v}(k)}\right\} R_{\ell, m}\left(\boldsymbol{\alpha}_{\mathfrak{u}} ; \boldsymbol{\beta}_{\mathfrak{v}}\right) .
$$

That is, $Z^{\nu_{1}, \nu_{2}} / Z$ is the generating function for the correlation functions of our microcanonical ensemble. 


\subsubsection{Expanding the Pfaffian Formulation of $Z^{\nu_{1}, \nu_{2}}$}

It is easily computed that

$$
\begin{aligned}
\langle f \mid g\rangle_{1}^{\nu_{1}}=\langle f \mid g\rangle_{1}+2 \sum_{n=1}^{N} a_{n}\left[\widetilde{f}\left(x_{n}\right) \epsilon_{1} \widetilde{g}\left(x_{n}\right)-\widetilde{g}\left(x_{n}\right) \epsilon_{1} \widetilde{f}\left(x_{n}\right)\right] & \\
& -2 \sum_{n=1}^{N} \sum_{m=1}^{N} a_{n} a_{m} \widetilde{f}\left(x_{n}\right) \widetilde{g}\left(x_{m}\right) \frac{\operatorname{sgn}\left(x_{n}-x_{m}\right)}{2},
\end{aligned}
$$

and

$$
\langle f \mid g\rangle_{4}^{\nu_{2}}=\langle f \mid g\rangle_{4}+\sum_{n=1}^{N} b_{n}\left[\widetilde{f}\left(y_{n}\right) \epsilon_{2} \widetilde{g}\left(y_{n}\right)-\widetilde{g}\left(y_{n}\right) \epsilon_{2} \widetilde{f}\left(y_{n}\right)\right] .
$$

For convenience let us write

$$
\mathcal{E}_{1,1}(x, y)=\frac{1}{4} \operatorname{sgn}(y-x) \quad \text { and } \quad \mathcal{E}_{1,2}(x, y)=\mathcal{E}_{2,1}(x, y)=\mathcal{E}_{2,2}(x, y)=0,
$$

and define

$$
\begin{gathered}
i(n)=\left\{\begin{array}{rr}
1 & 1 \leq n \leq N \\
2 & N<n \leq 2 N
\end{array}\right. \\
c_{n}=\left\{\begin{array}{l}
2 a_{n} \quad 1 \leq n \leq N ; \\
b_{n-N} \quad N<n \leq 2 N,
\end{array} \quad \text { and } \quad z_{n}= \begin{cases}x_{n} & 1 \leq n \leq N \\
y_{n-N} & N<n \leq 2 N\end{cases} \right.
\end{gathered}
$$

so that,

$$
\begin{aligned}
\langle f \mid g\rangle_{1}^{\nu_{1}}+\langle f \mid g\rangle_{4}^{\nu_{2}}=\langle f \mid g\rangle_{1}+\langle f \mid g\rangle_{4} & +\sum_{n=1}^{2 N} c_{n}\left[\widetilde{f}\left(z_{n}\right) \epsilon_{i(n)} \widetilde{g}\left(z_{n}\right)-\widetilde{g}\left(z_{n}\right) \epsilon_{i(n)} \tilde{f}\left(z_{n}\right)\right] \\
& -\sum_{n=1}^{2 N} \sum_{m=1}^{2 N} c_{n} c_{m} \widetilde{f}\left(z_{n}\right) \widetilde{g}\left(z_{m}\right) \mathcal{E}_{i(m), i(n)}\left(z_{m}, z_{n}\right) .
\end{aligned}
$$

Defining $\mathbf{A}^{\mathbf{p}}$ and $\mathbf{B}^{\mathbf{p}}$ as in Theorem 3.1 and

$$
\mathbf{A}^{\mathbf{p}, \nu_{1}}=\left[\left\langle p_{j} \mid p_{k}\right\rangle_{1}^{\nu_{1}}\right]_{j, k=1}^{N}, \quad \mathbf{B}^{\mathbf{p}, \nu_{1}}=\left[\left\langle p_{j} \mid p_{k}\right\rangle_{1}^{\nu_{1}}\right]_{j, k=1}^{N},
$$

we immediately see that

$$
\mathbf{A}^{\mathbf{p}, \nu_{1}}+\mathbf{B}^{\mathbf{p}, \nu_{2}}=\underbrace{\mathbf{C}^{\mathbf{p}}}_{=\mathbf{A}^{\mathbf{p}+\mathbf{B}^{\mathbf{p}}}}+\mathbf{W}^{\mathbf{p}},
$$

where the $j, k$ entry of $\mathbf{W}^{\mathbf{p}}$ is given by

$$
\begin{aligned}
\sum_{n=1}^{2 N} c_{n}\left[\widetilde{p}_{j}\left(z_{n}\right) \epsilon_{i(n)} \widetilde{p}_{k}\left(z_{n}\right)\right. & \left.-\widetilde{p}_{k}\left(z_{n}\right) \epsilon_{i(n)} \widetilde{p}_{j}\left(z_{n}\right)\right] \\
& -\sum_{n=1}^{2 N} \sum_{m=1}^{2 N} c_{n} c_{m} \widetilde{p}_{j}\left(z_{n}\right) \widetilde{p}_{k}\left(z_{m}\right) \mathcal{E}_{i(m), i(n)}\left(z_{m}, z_{n}\right) .
\end{aligned}
$$

Next we define the $N \times 4 N$ matrix $\mathbf{X}$ by

$$
\mathbf{X}=\left[\sqrt{c_{m}} \widetilde{p}_{j}\left(z_{m}\right) \quad \sqrt{c_{m}} \epsilon_{i(m)} \widetilde{p}_{j}\left(z_{m}\right)\right] \quad j=0, \ldots N-1 ; \quad m=1, \ldots, 2 N .
$$


We also define the $4 N \times 4 N$ matrix $\mathbf{J}$ by

$$
\mathbf{J}=\left[\begin{array}{ccccc}
0 & 1 & & & \\
-1 & 0 & & & \\
& & \ddots & & \\
& & & 0 & 1 \\
& & & -1 & 0
\end{array}\right]
$$

and the $4 N \times 4 N$ matrix $\mathbf{Y}$ by

$$
\mathbf{Y}=-\mathbf{J}+\left[\begin{array}{cc}
\sqrt{c_{m} c_{n}} \mathcal{E}_{i(m), i(n)}\left(z_{m}, z_{n}\right) & 0 \\
0 & 0
\end{array}\right]_{m, n=1}^{2 N} .
$$

Finally, we set $\mathbf{Z}=\left(\mathbf{C}^{\mathbf{p}}\right)^{-\mathrm{T}}=\left[\zeta_{j, k}\right]_{j, k=0}^{N-1}$.

A bit of matrix algebra reveals that

$$
\mathbf{A}^{\mathbf{p}, \nu_{1}}+\mathbf{B}^{\mathbf{p}, \nu_{2}}=\mathbf{Z}^{-\boldsymbol{\top}}+\mathbf{X Y} \mathbf{X}^{\top}
$$

and therefore,

$$
\frac{Z^{\nu_{1}, \nu_{2}}}{Z}=\frac{\operatorname{Pf}\left(\mathbf{Z}^{-\boldsymbol{\top}}-\mathbf{X Y} \mathbf{X}^{\top}\right)}{\operatorname{Pf}\left(\mathbf{Z}^{-\boldsymbol{\top}}\right)}
$$

This is useful, since by the Pfaffian Cauchy-Binet identity [9, 4],

$$
\frac{Z^{\nu_{1}, \nu_{2}}}{Z}=\frac{\operatorname{Pf}\left(\mathbf{Y}^{-\mathbf{T}}-\mathbf{X}^{\top} \mathbf{Z X}\right)}{\operatorname{Pf}\left(\mathbf{Y}^{-\boldsymbol{T}}\right)}
$$

A bit more matrix algebra reveals that

$$
\mathbf{X}^{\top} \mathbf{Z X}=\left[\begin{array}{cc}
\sqrt{c_{n} c_{m}} \sum_{j, k=0}^{N-1} \widetilde{p}_{j}\left(z_{m}\right) \zeta_{j, k} \widetilde{p}_{k}\left(z_{n}\right) & \sqrt{c_{n} c_{m}} \sum_{j, k=0}^{N-1} \widetilde{p}_{j}\left(z_{m}\right) \zeta_{j, k} \epsilon_{i(n)} \widetilde{p}_{k}\left(z_{n}\right) \\
\sqrt{c_{n} c_{m}} \sum_{j, k=0}^{N-1} \epsilon_{i(m)} \widetilde{p}_{j}\left(z_{m}\right) \zeta_{j, k} \widetilde{p}_{k}\left(z_{n}\right) & \sqrt{c_{n} c_{m}} \sum_{j, k=0}^{N-1} \epsilon_{i(m)} \widetilde{p}_{j}\left(z_{m}\right) \zeta_{j, k} \epsilon_{i(n)} \widetilde{p}_{k}\left(z_{n}\right)
\end{array}\right]_{m, n=1}^{2 N},
$$

And since

$$
\mathbf{Y}^{-\mathbf{T}}=-\mathbf{J}-\left[\begin{array}{lc}
0 & 0 \\
0 & \sqrt{c_{m} c_{n}} \mathcal{E}_{i(m), i(n)}\left(z_{m}, z_{n}\right)
\end{array}\right]_{m, n=1}^{2 N},
$$

we have that $\operatorname{Pf}\left(\mathbf{Y}^{-\mathbf{T}}\right)=(-1)^{N}$. This implies that

$$
\frac{Z^{\nu_{1}, \nu_{2}}}{Z}=\operatorname{Pf}\left(\mathbf{X}^{\top} \mathbf{Z X}-\mathbf{Y}^{-\mathbf{\top}}\right)
$$


Looking at the entries of $\mathbf{X}^{\top} \mathbf{Z X}$ and using the definitions of $\varkappa_{N}, \epsilon_{1}$ and $\epsilon_{2}$, we see

$$
\begin{aligned}
\sum_{j, k=0}^{N-1} \widetilde{p}_{j}\left(z_{m}\right) \zeta_{j, k} \widetilde{p}_{k}\left(z_{n}\right) & =\varkappa_{N}\left(z_{m}, z_{n}\right) \\
\sum_{j, k=0}^{N-1} \widetilde{p}_{j}\left(z_{m}\right) \zeta_{j, k} \epsilon_{i(n)} \widetilde{p}_{k}\left(z_{n}\right) & =\varkappa_{N} \epsilon_{i(n)}\left(z_{m}, z_{n}\right) \\
\sum_{j, k=0}^{N-1} \epsilon_{i(m)} \tilde{p}_{j}\left(z_{m}\right) \zeta_{j, k} \widetilde{p}_{k}\left(z_{n}\right) & =\epsilon_{i(m)} \varkappa_{N}\left(z_{m}, z_{n}\right) \\
\sum_{j, k=0}^{N-1} \epsilon_{i(m)} \tilde{p}_{j}\left(z_{m}\right) \zeta_{j, k} \epsilon_{i(n)} \tilde{p}_{k}\left(z_{n}\right) & =\epsilon_{i(m)} \varkappa_{N}\left(z_{m}, z_{n}\right) \epsilon_{i(n)} .
\end{aligned}
$$

It follows that

$$
\frac{Z^{\nu_{1}, \nu_{2}}}{Z}=(-1)^{N} \operatorname{Pf}(-\mathbf{J}-\mathbf{K})=\operatorname{Pf}(\mathbf{J}+\mathbf{K})
$$

where

$$
\mathbf{K}=\left[\sqrt{c_{m} c_{n}} K_{N}^{i(m), i(n)}\left(z_{m}, z_{n}\right)\right]_{m, n=1}^{2 N}
$$

Using these definitions and the formula for the Pfaffian of the sum $\mathbf{J}+\mathbf{K}$, [10, we find that

$$
\frac{Z^{\nu_{1}, \nu_{2}}}{Z}=\sum_{n=1}^{2 N} \sum_{\mathfrak{t}: \underline{\underline{n}} \nearrow \underline{2 N}} \operatorname{Pf} \mathbf{K}_{\mathfrak{t}}
$$

where $\mathbf{K}_{\mathfrak{t}}$ is the $2 n \times 2 n$ antisymmetric matrix given by

$$
\mathbf{K}_{\mathfrak{t}}=\left[\sqrt{c_{\mathfrak{t}(j)} c_{\mathfrak{t}(k)}} K_{N}^{i \circ \mathfrak{t}(j), i \circ \mathfrak{t}(k)}\left(z_{\mathfrak{t}(j)}, z_{\mathfrak{t}(k)}\right)\right]_{j, k=1}^{n}
$$

For each $\mathfrak{t}: \underline{n} \nearrow \underline{2 N}$ there exists non-negative integers $\ell$ and $m$ such that $\ell+m=n$ and functions $\mathfrak{u}: \underline{\ell} \nearrow \underline{N}$ and $\mathfrak{v}: \underline{m} \nearrow \underline{N}$ given by

$$
\mathfrak{u}(j)=\mathfrak{t}(j) \quad \text { for all } j \text { such that } \mathfrak{t}(j) \leq N
$$

and

$$
\mathfrak{v}(j)=\mathfrak{t}(j)-N \quad \text { for all } j \text { such that } N<\mathfrak{t}(j) \leq 2 N .
$$

In this situation we write $\mathfrak{t}=\mathfrak{u} \vee \mathfrak{v}$. (We allow for the possibility that $\mathfrak{u}$ or $\mathfrak{v}$ is the "empty" function, in which case $\mathfrak{u} \vee \mathfrak{v}=\mathfrak{v}$ or $\mathfrak{u} \vee \mathfrak{v}=\mathfrak{u}$ respectively.) It follows that we may write

$$
\frac{Z^{\nu_{1}, \nu_{2}}}{Z}=\sum_{\ell=0}^{N} \sum_{m=0}^{N} \sum_{\mathfrak{u}: \underline{\ell} \nearrow \underline{\underline{N}} \mathfrak{v}: \underline{\underline{m} \nearrow \underline{N}}} \operatorname{Pf} \mathbf{K}_{\mathfrak{u} \vee \mathfrak{v}}
$$

Finally,

$$
\operatorname{Pf} K_{\mathfrak{u} \vee \mathfrak{v}}=\left\{2^{\ell} \prod_{j=1}^{\ell} a_{\mathfrak{u}(j)} \prod_{k=1}^{m} b_{\mathfrak{v}(k)}\right\} \operatorname{Pf}\left[\begin{array}{ll}
K_{N}^{1,1}\left(x_{\mathfrak{u}(j)}, x_{\mathfrak{u}\left(j^{\prime}\right)}\right) & K_{N}^{1,2}\left(x_{\mathfrak{u}(j)}, y_{\mathfrak{v}\left(k^{\prime}\right)}\right) \\
K_{N}^{2,1}\left(y_{\mathfrak{v}(k)}, x_{\mathfrak{u}\left(j^{\prime}\right)}\right) & K_{N}^{2,2}\left(y_{\mathfrak{v}(k)}, y_{\mathfrak{v}\left(k^{\prime}\right)}\right)
\end{array}\right]_{j, j^{\prime}, k, k^{\prime}=1}^{\ell, \ell, m, m}
$$


and thus,

$$
\begin{aligned}
& \frac{Z^{\nu_{1}, \nu_{2}}}{Z}=\sum_{\ell=0}^{N} \sum_{m=0}^{N} \sum_{\mathfrak{u}: \underline{\ell} \nearrow \underline{\underline{N}} \mathfrak{v}: \underline{\underline{m}} \nearrow \underline{\underline{N}}}\left\{\prod_{j=1}^{\ell} a_{\mathfrak{u}(j)} \prod_{k=1}^{m} b_{\mathfrak{v}(k)}\right\} \\
& \times 2^{\ell} \operatorname{Pf}\left[\begin{array}{ll}
K_{N}^{1,1}\left(x_{\mathfrak{u}(j)}, x_{\mathfrak{u}\left(j^{\prime}\right)}\right) & K_{N}^{1,2}\left(x_{\mathfrak{u}(j)}, y_{\mathfrak{v}\left(k^{\prime}\right)}\right) \\
K_{N}^{2,1}\left(y_{\mathfrak{v}(k)}, x_{\mathfrak{u}\left(j^{\prime}\right)}\right) & K_{N}^{2,2}\left(y_{\mathfrak{v}(k)}, y_{\mathfrak{v}\left(k^{\prime}\right)}\right)
\end{array}\right]_{j, j^{\prime}, k, k^{\prime}=1}^{\ell, m, m}
\end{aligned}
$$

Comparing the coefficient of $a_{1} a_{2} \cdots a_{\ell} b_{1} b_{2} \cdots b_{m}$ in this expression with that in (4.22) we find that

$$
R_{\ell, m}(\mathbf{x} ; \mathbf{y})=2^{\ell} \operatorname{Pf}\left[\begin{array}{ll}
K_{N}^{1,1}\left(x_{j}, x_{j^{\prime}}\right) & K_{N}^{1,2}\left(x_{j}, y_{k}\right) \\
K_{N}^{2,1}\left(y_{k}, x_{j^{\prime}}\right) & K_{N}^{2,2}\left(y_{k}, y_{k^{\prime}}\right)
\end{array}\right]_{j, j^{\prime}, k, k^{\prime}=1}^{\ell, \ell, m, m}
$$

as desired.

\subsection{Proof of Theorem 3.4}

Let $H_{n}$ be the standard Hermite polynomial. It is known (cf. [1])

$$
\left\langle H_{2 m}, H_{2 n+1}-4 n H_{2 n-1}\right\rangle_{1}=4 h_{2 n} \delta_{m, n},
$$

where

$$
h_{n}:=\int_{\mathbb{R}} H_{n}^{2}(x) e^{-x^{2}} d x=\sqrt{\pi} 2^{n} n ! .
$$

Using the fact that $H_{k}^{\prime}(x)=2 k H_{k-1}(x)$, it follows readily that

$$
\begin{aligned}
\left\langle H_{2 m}, H_{2 n+1}\right\rangle_{4} & =\int_{\mathbb{R}}\left(H_{2 m}(x) H_{2 n+1}^{\prime}(x)-H_{2 m}^{\prime}(x) H_{2 n+1}(x)\right) d x \\
& =2(2 n+1) h_{n} \delta_{m, n}-4 m h_{2 m-1} \delta_{m, n+1} \\
& =h_{2 n+1} \delta_{m, n}-h_{2 n+1} \delta_{m, n+1} .
\end{aligned}
$$

We look for skew orthogonal polynomials in the form of

$$
P_{2 m}(x)=\sum_{k=0}^{m} a_{k} H_{2 k}(x), \quad a_{0}=1,
$$

and determine the coefficients $a_{k}, 1 \leq k \leq m$, by the orthogonal conditions

$$
\left\langle P_{2 m}, H_{2 k+1}-4 k H_{2 k-1}\right\rangle=0, \quad k=0,1, \ldots, m-1 .
$$

This gives, by (4.24) and (4.25), the following equations on $a_{k}$,

$$
-h_{2 k} a_{k-1}+\left[h_{2 k+1}+4\left(k+X^{2}\right) h_{2 k}\right] a_{k}-h_{2 k+2} a_{k+1}=0, \quad k=0,1, \ldots, m-1,
$$

where we define $a_{-1}=0$. Rescale by setting $\widehat{a}_{k}:=\frac{k !}{(2 k) !} a_{k}$, the above equations become

$$
-(k+1) \widehat{a}_{k+1}+\left(2 k+\frac{1}{2}-X^{2}\right) \widehat{a}_{k}-\left(k+\frac{1}{2}\right) \widehat{a}_{k-1}, \quad k=0,1, \ldots, m-1,
$$


which can be used to determine $\widehat{a}_{k}$ recursively, starting from $\widehat{a}_{-1}=0$ and $\widehat{a}_{0}=1$. It turns out, however, that this is precisely the three-term recurrence relation for $L_{k}^{-\frac{1}{2}}\left(-X^{2}\right)$. Consequently, $\widehat{a}_{k}=L_{k}^{-\frac{1}{2}}\left(-X^{2}\right)$. Hence, we conclude

$$
P_{2 m}(x)=\sum_{k=0}^{m} a_{k} H_{2 k}(x), \quad a_{k}=\frac{k !}{(2 k) !} L^{-\frac{1}{2}}\left(-X^{2}\right) .
$$

The Hermite polynomials are related to the Laguerre polynomials by [11, p. 106]

$$
H_{2 k}(x)=(-1)^{k} 2^{2 k} k ! L_{k}^{-1 / 2}\left(x^{2}\right), \quad H_{2 k+1}(x)=(-1)^{k} 2^{2 k+1} k ! x L_{k}^{-1 / 2}\left(x^{2}\right) .
$$

Using this relation and the facts that

$$
L_{k}^{\alpha}(0)=\left(\begin{array}{c}
k+\alpha \\
k
\end{array}\right) \quad \text { and } \quad(2 k) !=\frac{2^{2 k}}{\sqrt{\pi}} \Gamma\left(k+\frac{1}{2}\right) \Gamma(k+1),
$$

we see that (4.26) becomes (3.3). Since $P_{2 m}$ is determined by (4.24) and (4.25), the same process shows that

$$
P_{2 m+1}(x)=\sum_{k=0}^{m} a_{k}\left(H_{2 k+1}(x)-4 k H_{2 k}(x)\right), \quad a_{k}=\frac{k !}{(2 k) !} L^{-\frac{1}{2}}\left(-X^{2}\right) .
$$

Using the fact that $H_{2 j}^{\prime}(x)=4 j H_{2 j-1}(x)$ and $H_{2 j+1}(x)=2 x H_{2 j}(x)-H_{2 j}^{\prime}(x)$, we see that

$$
P_{2 m+1}(x)=\sum_{k=0}^{m} a_{k}\left(2 x H_{2 k}(x)-2 H_{2 k}^{\prime}(x)\right)=2 x P_{2 m}(x)-2 P_{2 m}^{\prime}(x) .
$$

It remains to compute $\left\langle P_{2 m}, P_{2 m+1}\right\rangle$. By (4.24) and (4.25), we have

$$
\begin{aligned}
\left\langle P_{2 m}, P_{2 m+1}\right\rangle & =a_{m}\left\langle P_{2 m}, H_{2 m+1}-4 m H_{2 m-1}\right\rangle \\
& =a_{m}\left[-h_{2 m} a_{m-1}+\left(h_{2 m+1}+4\left(m+X^{2}\right) h_{2 m}\right) a_{2 m}\right] \\
& =a_{m} \sqrt{\pi} 2^{m+1} m !\left[-(2 m-1) L_{m-1}^{-\frac{1}{2}}\left(-X^{2}\right)+\left(4 m+2 X^{2}+1\right) L_{m}^{-\frac{1}{2}}\left(-X^{2}\right)\right] \\
& =a_{m} \sqrt{\pi} 2^{m+1} m ! 2(m+1) L_{m+1}^{-\frac{1}{2}}\left(-X^{2}\right),
\end{aligned}
$$

where the last step follows from the three-term relation of the Laguerre polynomials, from which (3.5) follows readily.

Finally, we turn to the proof of (3.4). Using $\frac{d}{d x} L_{k}^{\alpha}(x)=-L_{k+1}^{\alpha+1}(x)$ and $L_{k}^{\alpha}(x)=$ $L_{k}^{\alpha+1}(x)-L_{k-1}^{\alpha}(x)([11$, p. 102]), (3.3) gives

$$
\begin{aligned}
& P_{2 m+1}(x)=2 x \sum_{k=0}^{m}(-1)^{k} \widehat{L}_{k}^{-\frac{1}{2}}\left(-X^{2}\right)\left[L_{k}^{\frac{1}{2}}\left(x^{2}\right)+L_{k-1}^{\frac{1}{2}}\left(x^{2}\right)\right] \\
& =2 x\left[\sum_{k=0}^{m-1}(-1)^{k} L_{k}^{\frac{1}{2}}\left(x^{2}\right)\left(\widehat{L}_{k}^{-\frac{1}{2}}\left(-X^{2}\right)-\widehat{L}_{k+1}^{-\frac{1}{2}}\left(-X^{2}\right)\right)+(-1)^{m} \widehat{L}_{m}^{-\frac{1}{2}}\left(-X^{2}\right) L_{m}^{\frac{1}{2}}\left(x^{2}\right)\right],
\end{aligned}
$$

where $\widehat{L}_{k}^{\alpha}(x):=L_{k}^{\alpha}(x) / L_{k}^{\alpha}(0)$, the formula (3.4) then follows from

$$
x \widehat{L}_{k}^{\alpha}(x)=-(\alpha+1)\left(\widehat{L}_{k}^{\alpha}(x)-\widehat{L}_{k-1}^{\alpha}(x)\right),
$$

which is a rescaling of the identity $x L_{k}^{\alpha+1}(x)=-(k+1) L_{k+1}^{\alpha}(x)+(k+1+\alpha) L_{k}^{\alpha}(x)([11$, p. 102]). 


\title{
References
}

[1] M. Adler, P. J. Forrester, T. Nagao, and P. van Moerbeke. Classical skew orthogonal polynomials and random matrices. J. Statist. Phys., 99(1-2):141-170, 2000.

[2] G. Ben Arous and A. Guionnet. Large deviations for Wigner's law and Voiculescu's non-commutative entropy. Probab. Theory Related Fields, 108(4):517-542, 1997.

[3] Gérard Ben Arous and Ofer Zeitouni. Large deviations from the circular law. ESAIM Probab. Statist., 2:123-134 (electronic), 1998.

[4] Alexei Borodin and Christopher D. Sinclair. The Ginibre ensemble of real random matrices and its scaling limits. Comm. Math. Phys., 291(1):177-224, 2009.

[5] David Borwein, Jonathan M. Borwein, and Richard E. Crandall. Effective Laguerre asymptotics. SIAM J. Numer. Anal., 46(6):3285-3312, 2008.

[6] Alan Edelman, Eric Kostlan, and Michael Shub. How many eigenvalues of a random matrix are real? J. Amer. Math. Soc., 7(1):247-267, 1994.

[7] Peter Forrester. Log-gases and Random Matrices. London Mathematical Society Monographs. Princeton University Press, 2010.

[8] Peter J. Forrester and Taro Nagao. Eigenvalue statistics of the real Ginibre ensemble. Phys. Rev. Lett., 99, 2007.

[9] Eric M. Rains. Correlation functions for symmetrized increasing subsequences, 2000.

[10] John R. Stembridge. Nonintersecting paths, Pfaffians, and plane partitions. Adv. Math., 83(1):96-131, 1990.

[11] Gábor Szegő. Orthogonal polynomials. American Mathematical Society, Providence, R.I., fourth edition, 1975. American Mathematical Society, Colloquium Publications, Vol. XXIII.

\author{
BRIAN RIDER \\ Department of Mathematics, University of Colorado, Boulder CO 80309 \\ email: brian.rider@colorado.edu \\ Christopher D. Sinclair \\ Department of Mathematics, University of Oregon, Eugene OR 97403 \\ email: csinclai@uoregon.edu \\ YUAN XU \\ Department of Mathematics, University of Oregon, Eugene OR 97403 \\ email: yuan@uoregon.edu
}

